\title{
Short-Term Electric Load and Price Forecasting Using Enhanced Extreme Learning Machine Optimization in Smart Grids
}

\author{
Aqdas Naz ${ }^{1}$, Muhammad Umar Javed ${ }^{1}$, Nadeem Javaid ${ }^{1, *(0)}$, Tanzila Saba ${ }^{2}$, Musaed Alhussein ${ }^{3}$ \\ and Khursheed Aurangzeb $3, *$ (i) \\ 1 Department of Computer Science, COMSATS University Islamabad, Islamabad 44000, Pakistan; \\ aqdasmalik17@gmail.com (A.N.); umarkhokhar1091@gmail.com (M.U.J.) \\ 2 College of Computer and Information Systems, Al Yamamah University, Riyadh 11512, Saudi Arabia; \\ tsaba@psu.edu.sa \\ 3 Computer Engineering Department, College of Computer and Information Sciences, King Saud University, \\ Riyadh 11543, Saudi Arabia; musaed@ksu.edu.sa \\ * Correspondence: nadeemjavaid@comsats.edu.pk (N.J.); kaurangzeb@ksu.edu.sa (K.A.)
}

Received: 1 February 2019; Accepted: 22 February 2019; Published: 5 March 2019

check for updates

\begin{abstract}
A Smart Grid (SG) is a modernized grid to provide efficient, reliable and economic energy to the consumers. Energy is the most important resource in the world. An efficient energy distribution is required as smart devices are increasing dramatically. The forecasting of electricity consumption is supposed to be a major constituent to enhance the performance of SG. Various learning algorithms have been proposed to solve the forecasting problem. The sole purpose of this work is to predict the price and load efficiently. The first technique is Enhanced Logistic Regression (ELR) and the second technique is Enhanced Recurrent Extreme Learning Machine (ERELM). ELR is an enhanced form of Logistic Regression (LR), whereas, ERELM optimizes weights and biases using a Grey Wolf Optimizer (GWO). Classification and Regression Tree (CART), Relief-F and Recursive Feature Elimination (RFE) are used for feature selection and extraction. On the basis of selected features, classification is performed using ELR. Cross validation is done for ERELM using Monte Carlo and K-Fold methods. The simulations are performed on two different datasets. The first dataset, i.e., UMass Electric Dataset is multi-variate while the second dataset, i.e., UCI Dataset is uni-variate. The first proposed model performed better with UMass Electric Dataset than UCI Dataset and the accuracy of second model is better with UCI than UMass. The prediction accuracy is analyzed on the basis of four different performance metrics: Mean Absolute Percentage Error (MAPE), Mean Absolute Error (MAE), Mean Square Error (MSE) and Root Mean Square Error (RMSE). The proposed techniques are then compared with four benchmark schemes. The comparison is done to verify the adaptivity of the proposed techniques. The simulation results show that the proposed techniques outperformed benchmark schemes. The proposed techniques efficiently increased the prediction accuracy of load and price. However, the computational time is increased in both scenarios. ELR achieved almost $5 \%$ better results than Convolutional Neural Network (CNN) and almost 3\% than LR. While, ERELM achieved almost $6 \%$ better results than ELM and almost 5\% than RELM. However, the computational time is almost $20 \%$ increased with ELR and 50\% with ERELM. Scalability is also addressed for the proposed techniques using half-yearly and yearly datasets. Simulation results show that ELR gives 5\% better results while, ERELM gives $6 \%$ better results when used for yearly dataset.
\end{abstract}

Keywords: smart grid; forecasting; load; price; CNN; LR; ELR; RELM; ERELM 


\section{Introduction}

For electricity generation and distribution, Traditional Grids (TGs) are used. The infrastructure of TG is getting obsolete, which results in energy loss and less efficient output. Due to the usage of outdated infrastructure, intensive power losses are being faced. This intensive power loss leads to load shedding, which is one of the major issues of today's world [1]. TGs use fossil fuels like coal, petrol, diesel, etc., for the combustion process of turbines. The extensive use of fossil fuels lead to natural resource depletion and increase in pollution. The literature has suggested to use Renewable Energy Sources (RES) and to modify the existing TGs by incorporating the latest technologies and updated infrastructure to overcome these issues. The new and modified form of TG is the Smart Grid (SG) [2]. The Information and Communication Technology (ICT) is integrated with TG to make SG. It provides bi-directional communication between consumers and utility. It monitors, protects and optimizes the generation, distribution and consumption of electric energy. It incorporates the latest technologies in TG: technical, control and communication technologies, to enable efficient energy transmission. With an ever increasing dilemma of energy shortage and cost inflation, people are attracted towards the SG. It provides the consumers with a reliable, economical, sustainable, secure and efficient energy as it uses intelligent methods. In SG, Demand Side Management (DSM) is used, which encourages the consumers to efficiently optimize the energy usage. DSM allows efficient load utilization by shifting maximum load from on-peak hours to off-peak hours. Thus, it reduced the cost of electricity. The differences between TG and SG are summarized in Table 1 [3].

Table 1. Differences between TG and SG.

\begin{tabular}{cc}
\hline TG & SG \\
\hline Analogue & Digital \\
One way communication & Two way communication \\
Centralized power generation & Distributed power generation \\
Small number of sensors & Large number of sensors \\
Manual monitoring & Automatic monitoring \\
Difficult to locate failures & Easy to locate failures \\
\hline
\end{tabular}

Data analytics is the phenomenon of dealing with big data obtained from different sources. Big data is the term used for the datasets having large volume, velocity, variety and veracity. It has the problem of extreme complexity which makes the processing of data difficult. Data analytics techniques are the necessity for the processing of big data. Data analytics can be used in a number of fields. For example, handling the financial details of customers by a bank, dealing with the flight details of different passengers by an airline company, dealing with the electricity load and price forecasting of consumers, etc. In SG, data analytics is used to minimize the electricity cost and to improve the service quality of energy utilities. It is also used to predict the future patterns of electricity consumption. Forecasting is done to schedule the load consumption from on-peak hours to off-peak hours for next day, week or month to reduce the electricity cost and enhance user comfort [4].

The terms forecasting and prediction are used interchangeably in this article. The case with load and consumption is similar. The sole purpose of this work is to increase the accuracy of load and price forecasting. Two techniques are proposed to solve the aforementioned objectives, i.e., ELR and ERELM. Furthermore, two types of datasets are used, i.e., uni-variate and multi-variate. UCI is the uni-variate dataset. Uni-variate dataset contains one variable, i.e., load in this paper. However, real-time data has a number of variables. Thus, multi-variate dataset is required to handle multiple variables to achieve a better understanding. In this paper, multi-variate dataset, i.e., UMass Electric Dataset is used to predict the load and price. Two types of scenarios are considered in this paper, i.e., residential load and smart meters load. The proposed techniques outperformed existing techniques in terms of forecasting load and price. Consequently, energy prediction assists in energy management on the residential and 
utility side. List of abbreviations that are used in this paper is given in Table 2. Whereas, Table 3 shows complete list of symbols.

Table 2. List of abbreviations.

\begin{tabular}{|c|c|}
\hline Abbreviation & Full Form \\
\hline AEMO & Australia Electricity Market Operators \\
\hline AI & Artificially Intelligent \\
\hline ANN & Artificial Neural Network \\
\hline ARIMA & Auto Regressive Integrated Moving Average \\
\hline ARMAX & Auto Regressive Moving Average with Exogenous variables \\
\hline $\mathrm{BP}$ & Back Propagation \\
\hline CART & Classification and Regression Technique \\
\hline CNN & Convolutional Neural Network \\
\hline DAE & Deep Auto Encoders \\
\hline DE-SVM & Differential Evolution Support Vector Machine \\
\hline DNN & Deep Neural Network \\
\hline DRN & Deep Residual Network \\
\hline DSM & Demand Side Management \\
\hline DWT & Discrete Wavelet Transform \\
\hline ELM & Extreme Learning Machine \\
\hline EPEX & European Power Exchange \\
\hline ELR & Enhanced Logistic Regression \\
\hline ERELM & Enhanced Recurrent Extreme Learning Machine \\
\hline FFNN & Feed Forward Neural Network \\
\hline GCA & Gray Correlation Analysis \\
\hline GWO & Grey Wolf Optimization \\
\hline GRU & Gated Recurrent Unit \\
\hline ISO NECA & Independent System Operator New England Control Area \\
\hline KELM & Kernel Extreme Learning Machine \\
\hline KPCA & Kernel Principal Component Analysis \\
\hline LR & Logistic Regression \\
\hline LSTM & Long Short Term Memory \\
\hline MAE & Mean Absolute Error \\
\hline MAPE & Mean Absolute Percentage Error \\
\hline MISO & Midcontinent Independent System Operator \\
\hline MLP & Multi Layer Perceptron \\
\hline MLR & Multi Linear Regression \\
\hline MSE & Mean Square Error \\
\hline NLS-SVM & Nonlinear Least Square Support Vector Machine \\
\hline NN & Neural Network \\
\hline NYISO & New York Independent System Operator \\
\hline OS-ELM7 & Online Sequential Extreme Learning Machine \\
\hline PJM & Pennsylvania-New Jersey-Maryland \\
\hline PSO & Particle Swarm Optimization \\
\hline RBM & Restricted Boltzmann Machine \\
\hline RELM & Recurrent Extreme Learning Machne \\
\hline ReLU & Rectified Linear Unit \\
\hline RES & Renewable Energy Sources \\
\hline RFE & Recursive Feature Elimination \\
\hline RMSE & Root Mean Square Error \\
\hline RNN & Recurrent Neural Network \\
\hline SARIMA & Seasonal Auto Regressive Integrated Moving Average \\
\hline SBELM & Sparse Bayesian Extreme Learning Machine \\
\hline SDA & Stacked De-noising Autoencoders \\
\hline SG & Smart Grid \\
\hline SLFN & Single Layer Feedforward Network \\
\hline SM & Smart Meters \\
\hline TG & Traditional Grid \\
\hline TVC-ABC & Time Varying Coefficients Artificial Bee Colony \\
\hline
\end{tabular}


Table 3. List of symbols.

\begin{tabular}{cc}
\hline Symbol & Description \\
\hline $\mathrm{x}$ & Actual value \\
$\mathrm{x}^{\prime}$ & Predicted value \\
$\mathrm{t}$ & Time slot \\
$\mathrm{y}$ & Output \\
$\mathrm{h}$ & Step size \\
$\mathrm{m}$ & Mean \\
$A_{v}$ & Actual value \\
$F_{v}$ & Forecasted value \\
$\mathrm{T}$ & Total time duration \\
$\mathrm{N}$ & Total number of samples \\
$\alpha$ & Fittest wolf 1 \\
$\beta$ & Fittest wolf 2 \\
$\delta$ & Fittest wolf 3 \\
$\omega$ & Remaining wolves \\
\hline
\end{tabular}

The rest of the paper is organized as: Section 2 deals with related work, Section 3 contains the detailed description of techniques used in this paper. Section 4 covers the proposed system models. Results and their discussion are given in Section 5, whereas Section 6 consists of evaluation of the proposed models using the performance metrics. Conclusion and future studies are discussed in Section 7.

\subsection{Motivation}

The authors in [5] used Multi Layer Perceptron (MLP) and Artificial Neural Network (ANN) to solve the load and price forecasting problem. We proposed an enhanced technique to increase the accuracy of load and price forecasting based on a modified loss function. In Reference [6], authors used ELM and RELM to predict electricity load. We proposed an enhanced technique to optimize weights and biases of network for efficient load forecasting. Furthermore, two scenarios are considered and two different types of datasets are used to predict the load and price efficiently.

\subsection{Problem Statement}

Data of SGs are increasing dramatically so an efficient technique is required to predict the load and price of electricity. Authors in [6] used Recurrent Extreme Learning Machine (RELM) to predict the electricity load. However, in RELM, weights and biases are randomly assigned which leads to drastic variations in prediction results. An enhanced technique is proposed to solve the aforesaid issue. In Reference [7], authors used Convolutional Neural Networks (CNNs) for predicting the energy demand. However, $\mathrm{CNN}$ involves tuning of a number of layers which makes it spatio-temporal complex.

In this paper, two enhanced techniques are proposed to increase the accuracy of load and price of electricity efficiently. Uni-variate and multi-variate datasets are used for both techniques. Furthermore, analysis of both residential and utility data is performed collectively.

\subsection{Contributions}

The following are the contributions of this paper:

- Feature engineering is performed using Recursive Feature Elimination (RFE), Classification And Regression Technique (CART) and Relief-F

- Two new classification techniques are proposed, i.e., Enhanced Logistic Regression (ELR) and Enhanced Recurrent Extreme Learning Machine (ERELM)

- In ELR, the loss function of Logistic Regression (LR) is modified to increase the prediction accuracy 
- The Grey Wolf Optimizer (GWO) learning algorithm is used with Recurrent Extreme Learning Machine (RELM) to optimize weights and biases in order to improve the forecasting accuracy

- The proposed techniques predict the electricity load and price efficiently

- ELR is used to predict the load and price of a smart home, whereas ERELM is used for forecasting the load of smart meters

- Cross validation is performed using K-Fold and Monte Carlo methods for assigning the fixed optimal values to weights and biases. This further increases the efficiency of GWO

- The accuracy of the proposed techniques is evaluated using the performance metrics, i.e., Mean Absolute Error (MAE), Mean Square Error (MSE), Mean Absolute Percentage Error (MAPE) and Root Mean Square Error (RMSE)

\section{Related Work}

Many forecasting techniques have been used in the past for load and price forecasting. These techniques can be categorized in three main groups: data driven, classical and Artificial Intelligence (AI). Data driven techniques consider past data to predict the desired outcomes. Classical methods comprise of the statistical and mathematical methods like Autoregressive Integrated Moving Average (ARIMA), Seasonal Autoregressive Integrated Moving Average (SARIMA), Random Forest (RF), etc., whereas AI methods mimic the behaviour of biological neurons like Feed Forward Neural Network (FFNN), Convolutional Neural Network (CNN), Long Short Term Memory (LSTM), etc.

\subsection{Electricity Load Forecasting}

In Reference [8], behavioural analytics are performed using Bayesian network and Multi Layer Perceptron (MLP). A number of experiments were performed using the data obtainedfrom the smart meters. Both short-term and long-term forecasting was performed. In Reference [9], Multiple Linear Regression (MLR) is used for forecasting purpose. However, it has the limitation that it can not be used for long term prediction. The authors in [10] used residual network for forecasting load on the basis of weather data. The authors in [11] used Restricted Boltzmann Machine (RBM) to train the data and Rectified Linear Unit (ReLU) to predict the electricity load. In Reference [12], Discrete Wavelet Transform (DWT) and Inconsistency Rate (IR) methods are proposed to select the optimal features from the feature set which helps in dimensionality reduction. Sperm Whale Algorithm (SWA) helps to optimize the parameters of SVM. Authors in [13] proposed a model for Short Term Load Forecasting (STLF). Mutual Information (MI) is used for feature selection whereas, better forecasting results are achieved by modifying the Artificial Neural Network (ANN). In Reference [14], authors predicted $24 \mathrm{~h}$ ahead cooling load of buildings using deep learning. The results show that deep learning techniques enhanced the load prediction. Similarly in [15], authors used Recurrent Neural Network (RNN), which groups the consumers into pool of inputs. The proposed model is implemented using Tensorflow package and it achieved better results.

ELM is a generalized single hidden layer FFNN learning algorithm that is proposed by the authors in References $[16,17]$. It proved to be effective in both regression and classification methods. In References [18,19], authors used the NNs for achieving better load prediction. In ELM learning processes, input weights and biases are randomly assigned, whereas output weights are calculated using the Moore-Penrose generalized inverse technique. In Reference [20], authors used Sparse Bayesian ELM for multi-classification purposes. The authors in [21] used Particle Swarm Optimization (PSO) and Discrete Particle Swarm Optimization (DPSO) techniques for efficient load forecasting. Authors in [22] implemented GWO with NNs to optimize weights and biases. It is proved that optimization of weights and biases increases the efficiency of network. In References [23], ELM is trained using back propagation by using context neurons as input to hidden and input layers. Accuracy is improved by further adjusting weights using previous iteration errors, whereas biases and neurons selection affect prediction accuracy as already discussed in [6]. 


\subsection{Electricity Price Forecasting}

In Reference [24], different models are used for price forecasting. These models belong to the class of deep learning. Based on the simulation results, it is proved that deep learning models perform better than the statistical models. In this paper, Gated Recurrent Unit (GRU) is used which is a variant of RNN. GRU outperformed LSTM and many other statistical models in terms of accuracy. In Reference [25], price forecasting is done using a variant of Auto Regressive Moving Average Model (ARMAX), i.e., Hilbertian ARMAX which uses the exogenous variables. The functional parameters used are modeled as the linear combinations of the sigmoid functions. These parameters are then optimized using a Quasi Newton (QN) algorithm. In Reference [26], two AI networks: CNN and LSTM are used for price forecasting in PJM electricity market. In Reference [27], Deep Neural Network (DNN) is used to extract complex patterns from the price dataset of Belgium. In Reference [28], Gray Correlation Analysis (GCA) is used along with Kernel Principal Component Analysis (KPCA) to deal with the dimensionality reduction issue. For prediction, Support Vector Machine (SVM) is used in combination with Differential Evolution (DE), where DE is used to tune the parameters of SVM. In Reference [29], a variant of autoencoder is used. This method comprises of encoder and decoder. First, the data is encoded to deal with space complexity. Once the output is obtained, it is decoded into original form. The authors in [30] implemented an enhanced form of Artificial Bee Colony (ABC) known as Time Varying Coefficients Artifical Bee Colony (TVC-ABC) for parameter tuning of Nonlinear Least Square Support Vector Machine (NLS-SVM). Inputs are first fed to ARIMA and then the output of ARIMA is given to NLS-SVM. This ARIMA + TVC-ABC NLS-SVM is a Multi Input Multi Output (MIMO) forecast engine. Limitations of gradient decent methods led researchers to evolve ELM based upon local minima, learning rate, stopping condition and iterations of learning [31]. ELM performance is different from traditional learning algorithms because it gives comparatively less forecasting error as well as proposing better generalization performance in [32].

Different versions of ELM have also been proposed by researchers such as Kernel Based Extreme Learning Machine (KELM). Robust classification is done in this paper. It is inspired by Mercer condition [33]. Related work is summarized in Table 4. 
Table 4. Summary of related work.

\begin{tabular}{|c|c|c|c|c|}
\hline Technique & Features/DataSet & Region & Contributions & Limitations \\
\hline Bayesian, MLP [8] & Load/UKDale & UK & Forecasting done using behavioural analytic & Requires intensive training \\
\hline MLR, BaggedT, NN [9] & Load and weather/Beijing & Beijing, China & $\begin{array}{l}\text { Comparison between techniques done to } \\
\text { overcome the limitations }\end{array}$ & $\begin{array}{l}\text { Not suitable for long } \\
\text { term forecasting }\end{array}$ \\
\hline DRN [10] & Load and weather/ISO-NE & New England & Load forecasting done using weather data & Over fitting \\
\hline RBM, ReLU [11] & Load/Korea Electric Company & Korea & Two stage forecasting performed & $\begin{array}{l}\text { Long-term forecasting } \\
\text { not supported }\end{array}$ \\
\hline DWT-IR, SVM, SWA [12] & Load/NYISO, AEMO & Australia, US & $\begin{array}{l}\text { Dimensionality reduction and paramater } \\
\text { optimization done }\end{array}$ & Time complexity \\
\hline Modified MI, ANN [13] & Load/PJM & US & Two stage forecasting is done & Time complexity \\
\hline DAE [14] & Load/Hong Kong & Hong Kong & Cooling load prediction done & Time and space consuming \\
\hline Pooling Deep RNN [15] & Load/IRISH & Ireland & $\begin{array}{l}\text { Pooling of consumers done for } \\
\text { aggregated prediction }\end{array}$ & Difficult to train \\
\hline ELM $[16,17]$ & Load/USF & US & Long, medium and short term forecasting done & Over fitting \\
\hline SLFN $[18,19]$ & $\begin{array}{l}\text { Load/Marine Resources } \\
\text { Division }\end{array}$ & Australia & Optimization of weights & $\begin{array}{l}\text { Overfitting by using } \\
\text { moore-penrose inverse }\end{array}$ \\
\hline Sparse Bayesian ELM [20] & $\begin{array}{l}\text { Electricity Load/Harvard } \\
\text { medical college }\end{array}$ & USA & Optimization of weights and biases using BP & Require intensive training \\
\hline PSO, DPSO [21] & Load/US & USA & Compact ANNs are produced & Large computational time \\
\hline GWO with NN [22] & & USA & Weights and biases optimization & Time complexity \\
\hline RELM $[6,23]$ & $\begin{array}{l}\text { Electricity Load/Bench mark } \\
\text { UCI machine }\end{array}$ & Portugal & Use of context neurons & Computationally expensive \\
\hline LSTM, DNN, GRU [24] & Load and price/EPEX & Belgium & Comparison between different models & Over fitting \\
\hline Hilbertian, ARMAX [25] & Price/EPEX & Spain, Germany & $\begin{array}{l}\text { Optimization of functional parameters for } \\
\text { price forecasting }\end{array}$ & Non linearity \\
\hline CNN, LSTM [26] & Price/PJM & US & Two NNs are used for price forecasting & Computationally expensive \\
\hline
\end{tabular}


Table 4. Cont

\begin{tabular}{|c|c|c|c|c|}
\hline Technique & Features/DataSet & Region & Contributions & Limitations \\
\hline DNN [27] & Price/EPEX & Belgium & Complex patterns are extracted for prediction & Space complexity \\
\hline GCA, KPCA, DE-SVM [28] & Price/ISO NE-CA & New England & $\begin{array}{l}\text { Dimensionality reduction is removed using } \\
\text { hybrid of KPCA and GCA }\end{array}$ & Over fitting \\
\hline SDA [29] & Price/MISO & $\begin{array}{l}\text { Arkansas, Texas } \\
\text { and Indiana }\end{array}$ & Variant of autoencoder used & Computationally expensive \\
\hline $\begin{array}{l}\text { ARIMA, TVC-ABC, } \\
\text { NLS-SVM [30] }\end{array}$ & Price/PJM, NYISO, AEMO & Australia, US & Parameter tuning of SVM done using TV-ABC & Computationally expensive \\
\hline $\begin{array}{l}\text { ANN with meta heuristics } \\
\text { optimization methods [31] }\end{array}$ & $\begin{array}{l}\text { Load and price/Commercial } \\
\text { load of building in China, } \\
\text { Taiwan regional electricity load }\end{array}$ & Taiwan & $\begin{array}{l}\text { Various paramater calculations done } \\
\text { for accuracy }\end{array}$ & $\begin{array}{l}\text { Accuracy of models depend } \\
\text { on nature of dataset }\end{array}$ \\
\hline OS-ELM with kernel [32] & $\begin{array}{l}\text { Load and price/Sylva } \\
\text { bench mark }\end{array}$ & US & Comparison of different algorithms done & $\begin{array}{l}\text { Restrict to the computation } \\
\text { of streamed data }\end{array}$ \\
\hline $\begin{array}{l}\text { ELM in multi class } \\
\text { scenario [33] }\end{array}$ & $\begin{array}{l}\text { Load and price/University of } \\
\text { California Irvine }\end{array}$ & Canada & Robust classification & $\begin{array}{l}\text { Computational cost } \\
\text { overhead }\end{array}$ \\
\hline $\begin{array}{l}\text { Enhanced Logistic } \\
\text { Regression }\end{array}$ & $\begin{array}{l}\text { Load and Price/UMass } \\
\text { Electric Dataset }\end{array}$ & USA & ELR beats conventional techniques & Large computational time \\
\hline $\begin{array}{l}\text { RELM enhanced } \\
\text { using GWO }\end{array}$ & Load and Price/UCI Dataset & USA & $\begin{array}{l}\text { Optimized weights and biases leads to } \\
\text { better prediction }\end{array}$ & $\begin{array}{l}\text { Large computational time is } \\
\text { required for } \\
\text { weight optimization }\end{array}$ \\
\hline
\end{tabular}


In literature, short term load and price forecasting using the conventional techniques is performed on individual basis mostly, whereas we used short term load and price forecasting simultaneously using enhanced techniques which surpasses the conventional techniques in terms of accuracy. The first proposed technique, i.e., ELR outperformed LR in terms of prediction accuracy, whereas the second proposed technique ERELM outperformed ELM and RELM using GWO and performs much better due to weights and biases optimization.

\section{Existing and New Techniques}

In this section, the existing and the proposed techniques are discussed.

\subsection{Classification and Regression Technique (CART)}

CART is a type of decision tree algorithm which consists of both classification and regression procedures and is used to predict the continuous and discrete variables, respectively. CART uses historical data to build decision trees. These newly built trees are then used for classification of data. It is a binary recursive process. Binary process has only two output values, i.e., 0 and 1 . The algorithm will search for all possible values and variables before performing the split operation [34]. The CART method has three main parts:

- Construction of maximum tree,

- Choice of right tree size,

- Classification of data using the constructed tree.

The construction of a maximum tree refers to splitting of the tree till the last set of observations. This is the most time-consuming phase in CART. Constructing the maximum trees is a complex method which can have more than hundred levels. Therefore, the trees must be optimized before being used for classification of the data. The classification problems are the ones which involve discrimination between entities, e.g., discrimination among students to decide which student will be awarded with the degree this year. On the other hand, regression uses historical data patterns to predict the future values, e.g., load and price prediction of homes. The steps of CART are stated below:

- Problem definition,

- Variable selection,

- Specifying the accuracy criteria,

- Selecting split size,

- Determine the threshold to stop splitting,

- $\quad$ Selection of the best tree.

\subsection{Recursive Feature Elimination (RFE)}

RFE is a feature extraction process. It selects a set of most important features which are least redundant. As the name is self defining, it is an iterative process which keeps running in a loop unless all the best features are selected. The selected features are then ranked in the order they are being removed from the feature set. The computation time depends upon the number of features which need to be eliminated [35]. The pseudocode of RFE is given in Algorithm 1. 


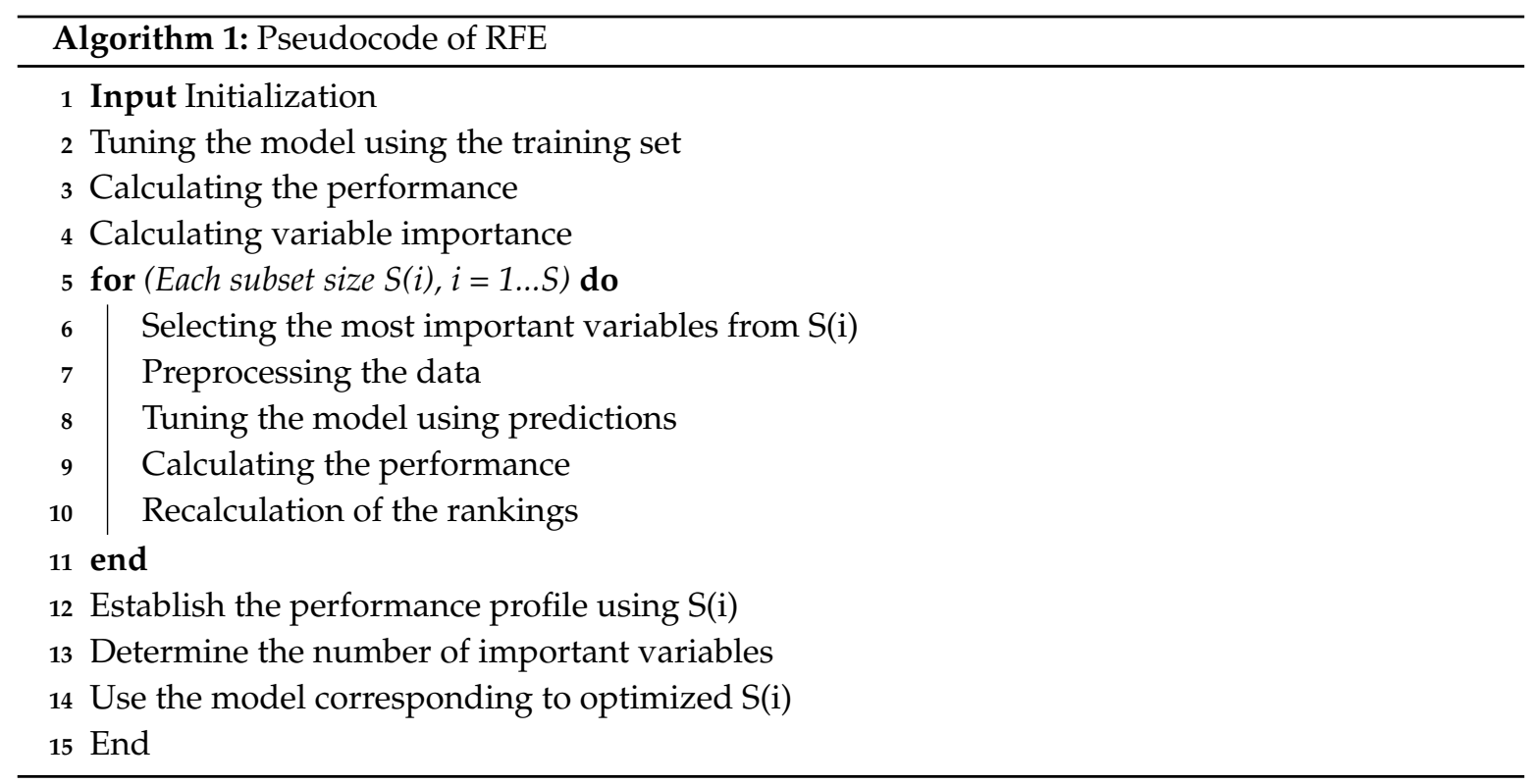

\subsection{Relief-F}

Relief-F is an extensively used method for feature selection. This method randomly selects an instance $\mathrm{R}$ and then find its nearest hits and miss instances. The nearest hits are the $k$-nearest neighbors of the selected random instance R. Afterwards, the average of all the weights of the nearest hits and miss is calculated to select the next instance. The pseudocode of Relief-F is discussed in Algorithm 2 [36].

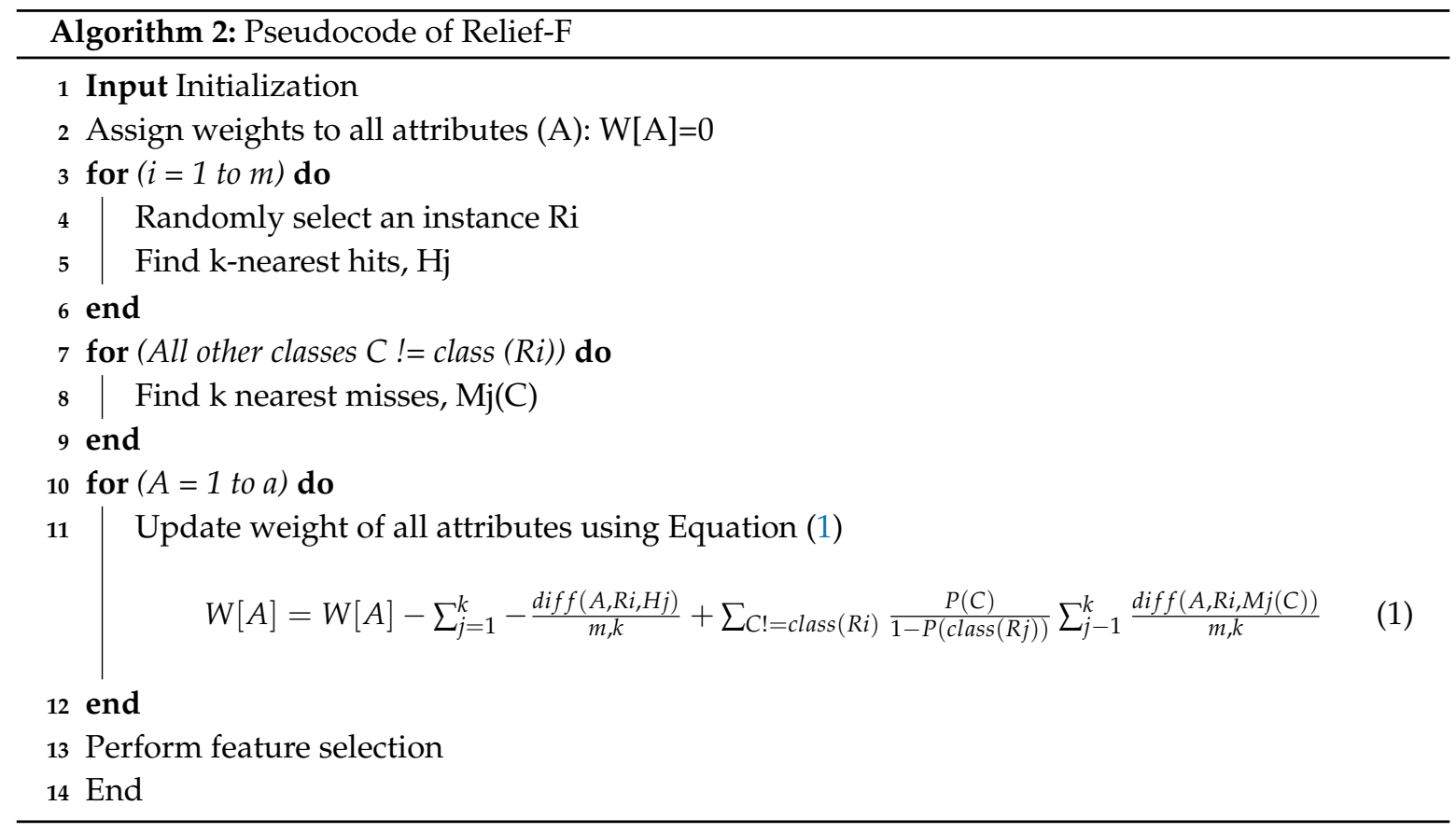

\subsection{Convolutional Neural Network (CNN)}

$\mathrm{CNN}$ is a type of NN. It is built from neurons and work like the biological neurons. Each neuron is fed with some input, and then it performs a dot product and finally gives the output. It consists of more than one convolutional layer; followed by a multilayer NN. The basic type of CNN is a 2D network and is mostly used for images. The layers in CNN are: pooling layer, dense layer, dropout layer and convolutional layer. For forecasting data, 1D CNN can also be used. It also has an activation 
function like Sigmoid, ReLU, Tanh, etc. When new inputs are given to CNN, it does not know the exact feature mapping. Therefore, it creates a convolutional layer and then convolves this layer to find the correct feature mapping. The pooling layer in CNN has the ability of shrinking the large inputs. The most widely used activation function for CNN is ReLU. Its working is simple; whenever a negative number occurs, it is replaced by 0 . Hidden layers are also present in CNN. The error minimization is performed using these layers.

\subsection{Logistic Regression (LR)}

LR is a type of statistical model used for regression. It is used to analyze a given dataset and then perform predictions using the independent variables. The outcome of LR is in the binary form. The main aim of LR is to describe a pattern between independent and dependent variables. There are two main parameters of LR: loss function and sigmoid function. The features should be in the binary form to use the LR method. Hence, normalization of data is required before implementing the LR model on the available data. The sigmoid function used in LR is given in following equation [37]:

$$
\operatorname{sigmoid}(t)=\frac{1}{1+e^{-t}}
$$

Logistic loss function is given by the following equation, which is taken from [37]:

$$
\text { lossfunction }(t)=\frac{1}{m}\left(-y^{t} \log (h)-(1-y)^{t} \log (1-h)\right) .
$$

\subsection{Enhanced Logistic Regression (ELR)}

ELR is proposed in this paper. It is an enhanced form of LR technique. In ELR, a new loss function is used. Loss function is a group of objective functions that need to be minimized. It is a measure of how good a prediction model performs in predicting the outcome. Minimizing the value of the loss function increases the prediction accuracy. In this paper, the loss function is being minimized to enhance the prediction accuracy. The equation for the loss function of ELR is given below:

$$
\text { newlossfunction }(t)=\frac{0.1}{m}\left(-y^{t} \log (h)-(1-y)^{t} \log (1-h)\right) .
$$

ELR is used to predict electricity load and price efficiently for a smart home and load of smart meters. Two different datasets, i.e., UMass Electric Dataset and UCI Dataset are used to test the proposed technique.

\subsection{Grey Wolf Optimizer (GWO)}

In this section, GWO technique is discussed in detail. In the proposed model, the metaheuristic technique GWO is used. It follows the social leadership and hunting mechanism of grey wolves as shown in Figure 1. The population is based on groups, i.e., alpha $(\alpha)$, beta $(\beta)$, gamma $(\gamma)$ and omega $(\omega) . \alpha, \beta$ and $\gamma$ are considered as the fittest wolves who guide other wolves $(\omega)$ in search space. Grey wolves update their location according to the positions of the three fittest wolves, i.e., $\alpha, \beta$ and $\gamma$ [22]. 


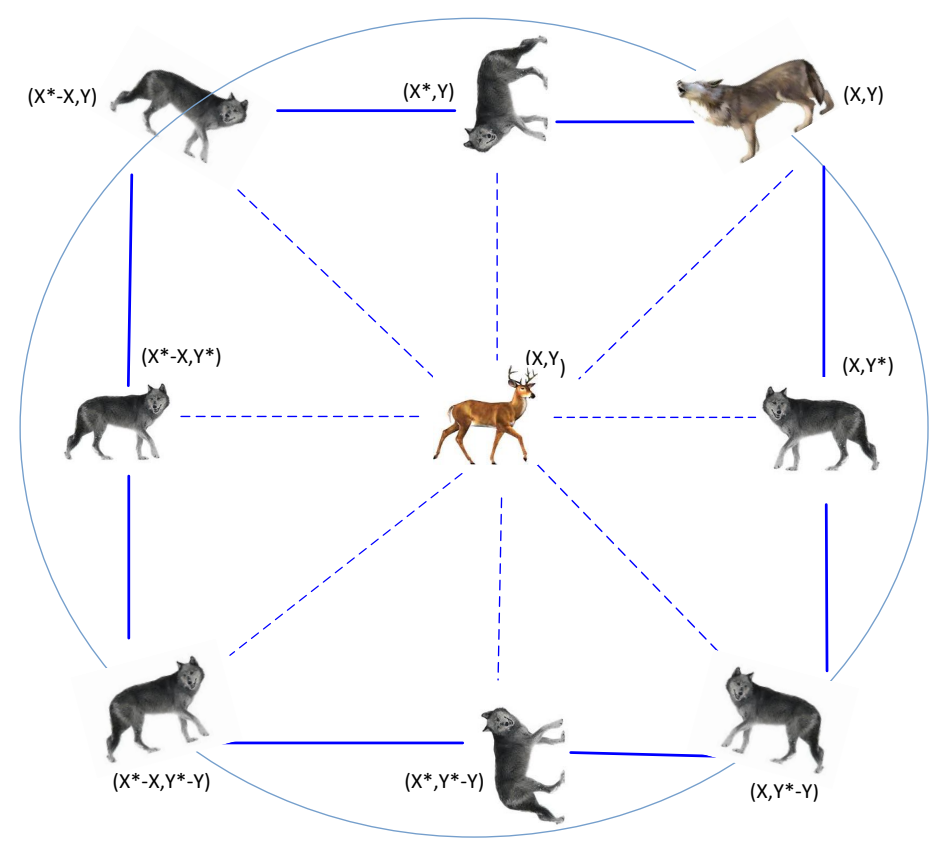

Figure 1. Grey wolf social hierarchy.

The general steps that are followed in GWO are:

- $\quad$ Parameters of grey wolves are initialized such as maximum number of iterations, the population size, upper and lower bounds of search space,

- Calculate fitness value to initialize the position of each wolf,

- $\quad$ Select three best wolves, i.e., $\alpha, \beta$ and $\gamma$,

- $\quad$ Calculate the positions of the remaining wolves $(\omega)$,

- Repeat from step 2 if current solution is not satisfied,

- The fittest solution is taken as $\alpha$.

The pseudocode of GWO is given in Algorithm 3.

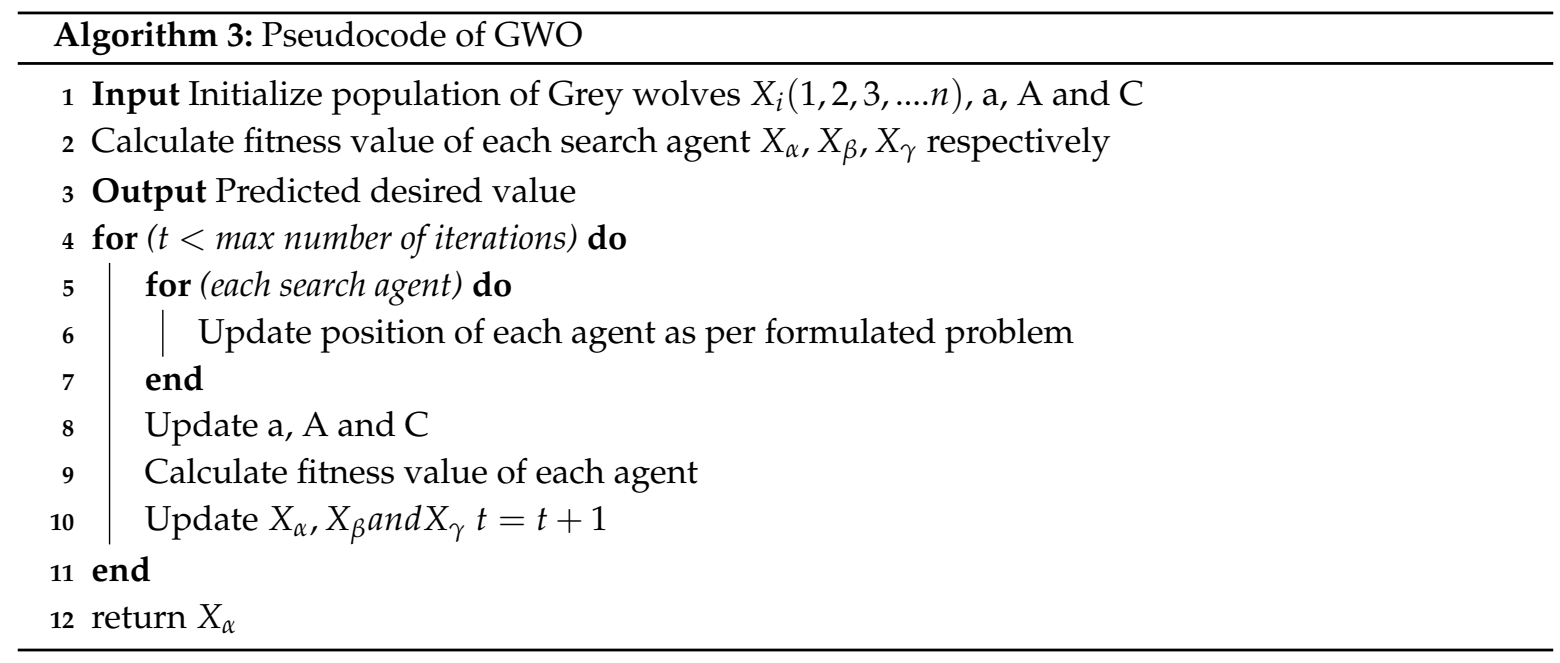




\subsection{Recurrent Extreme Learning Machine (RELM)}

RELM is a single hidden layer neural network (SHLRN). It is a feedback intra network that uses output or hidden layers as given in Equation (5) [6]:

$$
y=\sum_{j=1}^{m} \beta_{j} g\left(\sum_{i=1}^{n} w_{i, j} x_{i}+\sum_{n=i+1}^{n+r} W_{i, j} \delta(t-1+n)+b_{j}\right),
$$

where $\delta$ represents delay, $t$ shows current iteration and $r$ indicates total number of context neurons. Context neurons are connected backward from output to input. These neurons perform similar to input neurons and hold delayed values of output neurons. The learning method to update weights and biases of ELM and RELM is similar to that shown in Figure 2.

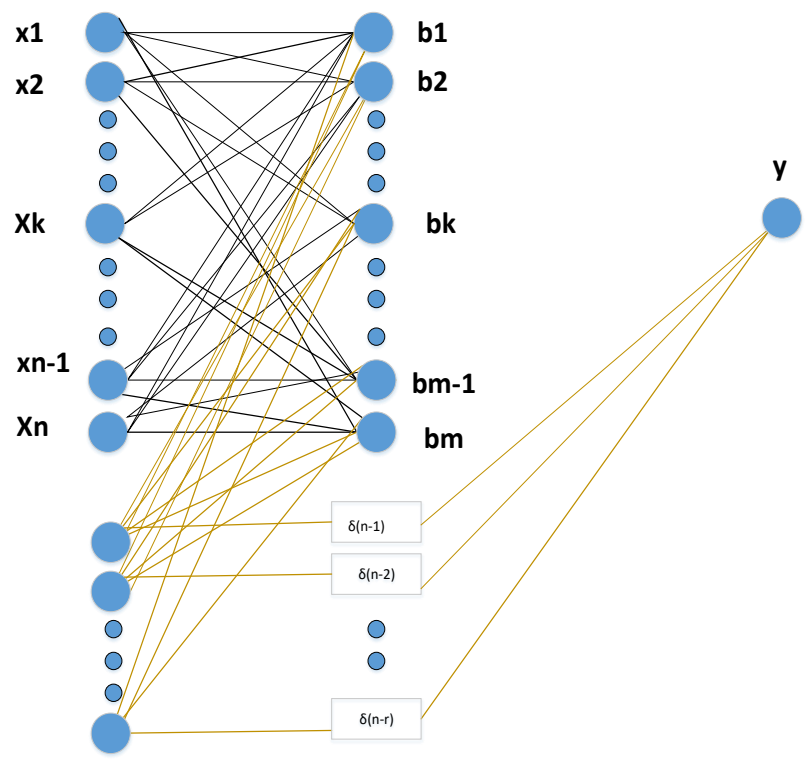

Figure 2. Functioning of RELM.

Weights and biases are decided randomly. Optimal results against weight and biases are utilized in RELM on a random basis. Training dataset is used to calculate the unknown weights of hidden layer. The unknown weights of hidden layer are calculated using a Moore-Penrose generalized inverse technique.

\subsection{Enhanced Recurrent Extreme Learning Machine (ERELM)}

ERELM is an enhanced form of RELM, whereas RELM is an enhanced form of ELM. ERELM is a single layer FFNN. In RELM, weights and biases are decided randomly, whereas the output weights are determined analytically. The output weights are determined using a simplified generalized inverse operation. The issue with ELM is that the classification boundary is not well defined and usually misclassifies some samples. To overcome this shortcoming, a new technique is proposed.

In the proposed technique, i.e., ERELM, weights and biases are decided after optimization using GWO algorithm. GWO finds the optimized solution which minimizes RMSE. Cross validation in ERELM is done using Monte Carlo and K-Fold methods. The Monte Carlo technique is used to model the probabilistic nature of the random variables. It performs risk analysis using the probability distribution. The common probability distributions used with Monte Carlo are: normal, uniform, triangular, discrete, etc. In K-Fold cross validation process, the entire dataset is divided into batches of $K$ samples. The value of $K$ could be any positive integer. Most commonly used $K$-Fold method is $10-$ Fold cross validation method, in which the value of $K$ is 10. Each batch formed after splitting of data in the validation process is termed as fold. The pseudocode of ERELM is discussed in Algorithm 4. 


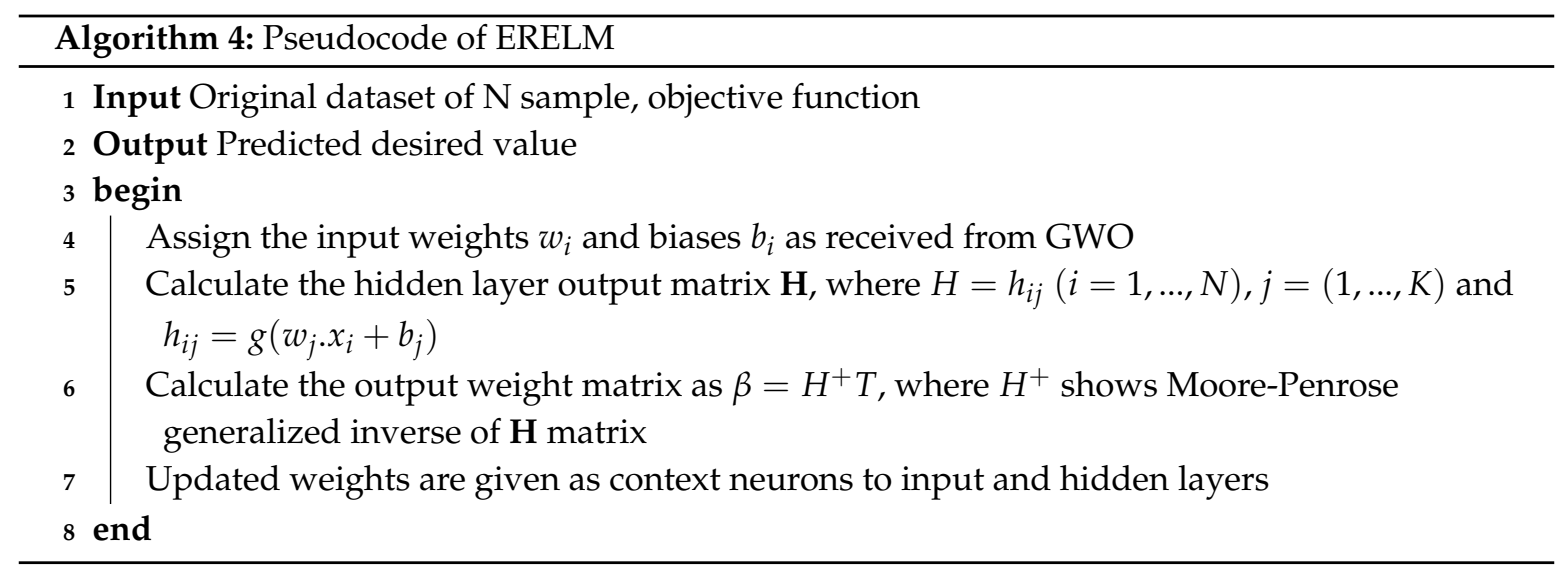

\section{Proposed System Models}

Two system models are proposed in this section. The description of these models are given below.

\subsection{Proposed System Model 1}

The proposed system model consists of residential load and price data of a SH. The SH under consideration consists of six rooms and eight heavy appliances. The proposed model consists of four basic steps, i.e., normalization of data, feature selection using CART and RFE, feature extraction using Relief-F and finally forecasting of load and price using CNN, LR and ELR. ELR is a proposed technique which outperformed CNN and LR in terms of prediction accuracy. In this model, short term forecasting is performed to make decisions for efficient load and price scheduling for the near future.

The first proposed model is shown in Figure 3.

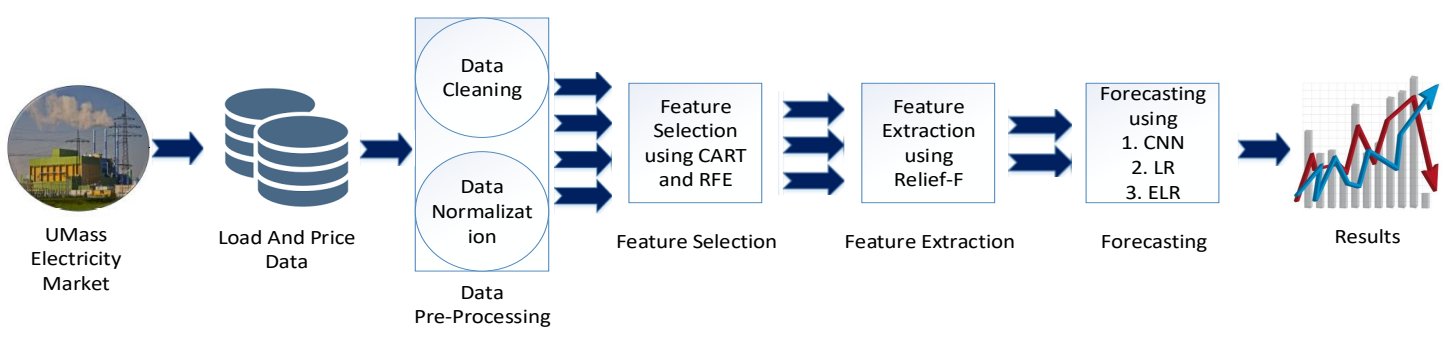

Figure 3. Proposed system model 1.

\subsection{Proposed System Model 2}

The second proposed system model is shown in Figure 4 . 


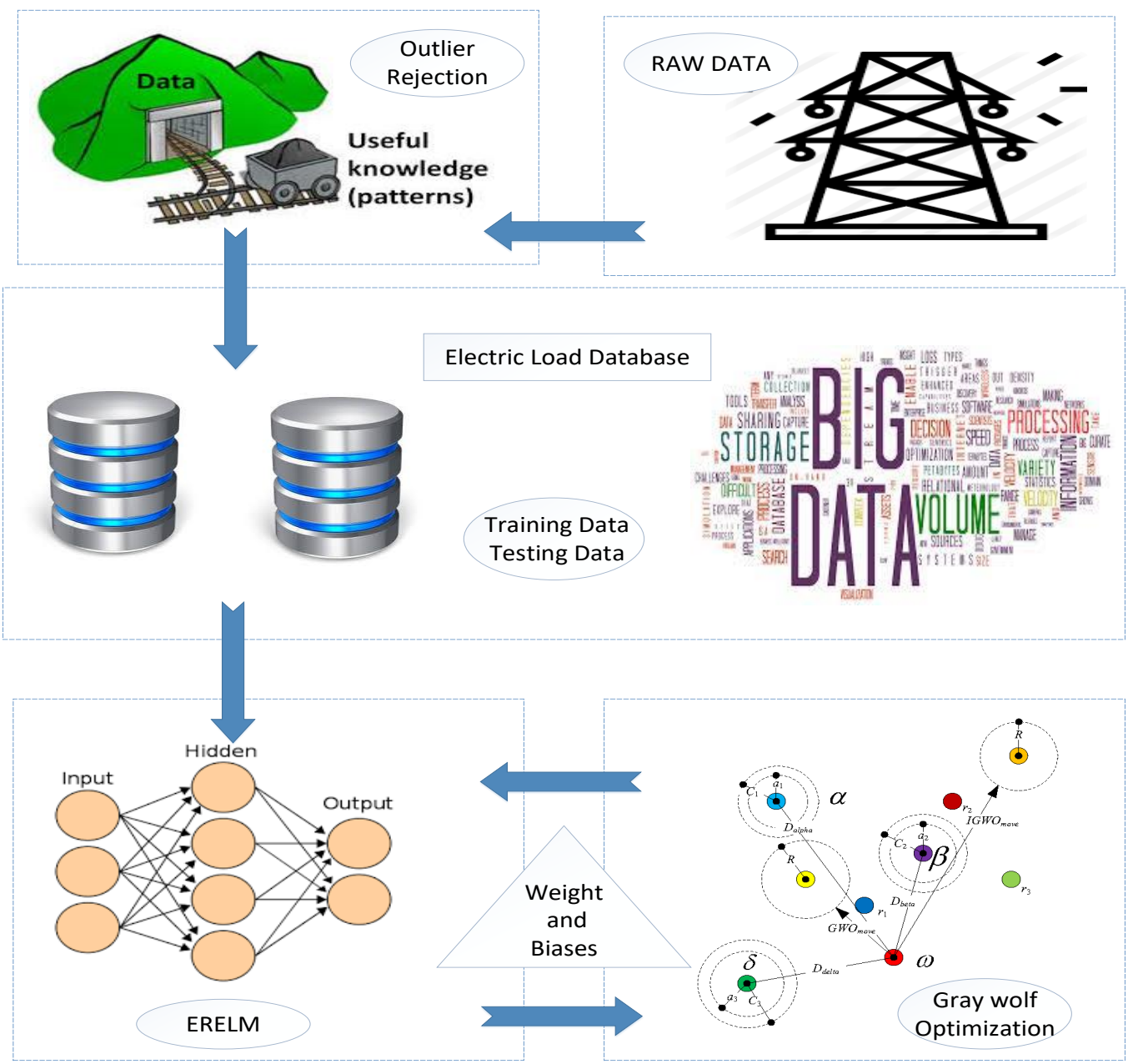

Figure 4. Proposed system model 2.

In the second system model, a load of 10 smart meters is taken. Subsequently, comparison is performed with multivariate residential data. The first step in this model is the preprocessing of data; after the data is preprocessed, the best parameters are selected using RELM. The optimization of RELM is performed using GWO. GWO optimizes biases and weights to improve the accuracy. Thereafter, the proposed technique ERELM reduces forecasting error. Cross validation is performed using Monte Carlo and K-Fold methods.

The simulation results and the assessment of both proposed models is done on the basis of four different performance metrics: MAPE, MAE, RMSE and MSE. The results show that the proposed techniques beat the existing techniques in terms of prediction accuracy.

\section{Simulation Results and Discussion}

This section covers the simulation results of the proposed models. The results are given in this section along with their discussion. The simulations are performed in Spyder (Python 3.6 package) provided by Anaconda (a data science platform manufactured by Anaconda, Inc. located in Austin, Texas, USA) on HP 450G ProBook, having 1 TB Hard Drive and 8 GB RAM.

\subsection{Simulation Results and Discussion of Proposed System Model 1}

The simulation results and discussion of proposed system model 1 is given below. 


\subsubsection{Data Description}

The first dataset is taken from UMass Electric Dataset [38]. It is a multivariate dataset used for forecasting purpose. Half-yearly and yearly data is taken for the year 2016 to address scalability. The dataset contains the half-hourly load and price values of a single home. The dataset is divided into a 70:30 ratio, i.e., seventy percent data is used for training, whereas the remaining thirty percent is used for testing. Preprocessing of the dataset is done to remove the Not a Number $(\mathrm{NaN})$ and blank values. UMass dataset is used for both proposed system models. Though, it performs much better when used with ELR.

Table 5 shows the features of UMass Electric Dataset excluding the target features. The targeted features are "Load" in case of load prediction and "Price" in case of price prediction. The values are given in standard units, i.e., $\mathrm{kW}$ for load and cents $/ \mathrm{kWh}$ for price. The dataset is normalized in the range [0-1].

Table 5. Features in UMass Electric Dataset.

\begin{tabular}{l}
\hline Original Features \\
\hline Air Conditioner (AC), Furnace, Cellar lights, Washer, First floor lights, Utility \\
room + Basement, Garage outlets, Master bed + Kids bed, Dryer, Panels, Home \\
office, Dining room, Microwave, Fridge
\end{tabular}

\subsubsection{CART}

Table 6 shows the results of CART used to predict load and price using UMass Electric Dataset. CART gives respective values for different features.

Table 6. Results of CART for UMass Electric Dataset.

\begin{tabular}{ccc}
\hline Features & Load Values & Price Values \\
\hline AC & 0.6653 & 0.6633 \\
Furnace & 0.0103 & 0.0101 \\
Cellar lights & 0.0018 & 0.0011 \\
Washer & 0.0029 & 0.0029 \\
First floor & 0.0032 & 0.0026 \\
Utility + Basement & 0.0615 & 0.0670 \\
Garage & 0.0036 & 0.0070 \\
M. bed + K. bed & 0.0080 & 0.0059 \\
Dryer & 0.1890 & 0.1927 \\
Panels & 0.0033 & 0.0030 \\
Home office & 0.0826 & 0.0083 \\
Dining room & 0.0079 & 0.0084 \\
Microwave & 0.0296 & 0.0262 \\
\hline
\end{tabular}

\subsubsection{RFE}

After using the CART technique, RFE is implemented for feature selection. RFE keeps on iterating unless model is left with only the most prominent features. The choice of features depend upon requirements. The results of RFE are given in Table 7.

RFE assigns two values to the features, i.e., True and False. In the proposed model, number of selected features through RFE is 8, when used for UMass Electric Dataset. The RFE selected features are: AC, cellar lights, washer, garage, master bed + kids bed, panels, dining room and microwave. For a UCI Dataset, RFE did not give any output as it is a uni-variate dataset. 
Table 7. RFE features for a UMass Electric Dataset.

\begin{tabular}{cc}
\hline Type & Number of Features \\
\hline Original & 16 \\
Selected & 8 \\
Rejected & 8 \\
\hline
\end{tabular}

\subsubsection{Relief-F}

Relief-F is used for feature extraction. The threshold for Relief-F is 10. Table 8 shows the Relief-F features for UMass Electric Dataset. It did not give any output when used with UCI Dataset because it is uni-variate.

Table 8. Relief-F features for UMass Electric Dataset.

\begin{tabular}{cc}
\hline Parameters & Values \\
\hline Threshold & 10 \\
Selected features & 5 \\
Nearest Neighbors & 3 \\
\hline
\end{tabular}

\subsubsection{Load Forecasting}

Figure $5 \mathrm{a}, \mathrm{b}$ show the load prediction comparison of three different techniques for one day using two different hourly datasets. Similarly, Figure $6 a, b$ show the load prediction comparison for one week using two different hourly datasets. From Figure 7a,b, monthly load prediction comparison can be observed. In this case, to avoid the cluttering of the graphs, data is taken after every four hours. These figures show that the proposed technique ELR outperformed LR and CNN for both datasets. It can be envisioned that the load prediction with ELR is close to the actual data. The prediction results obtained using a UMass Electric Dataset are better than the UCI Dataset.

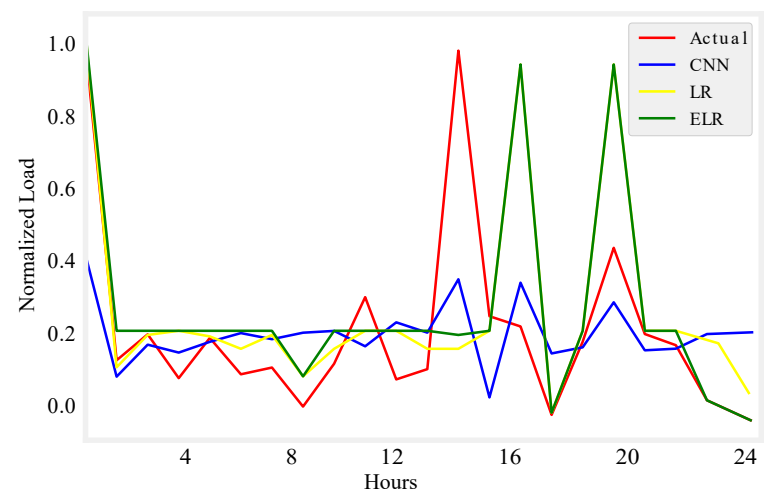

(a) Load prediction using UMass

Figure 5. Cont. 


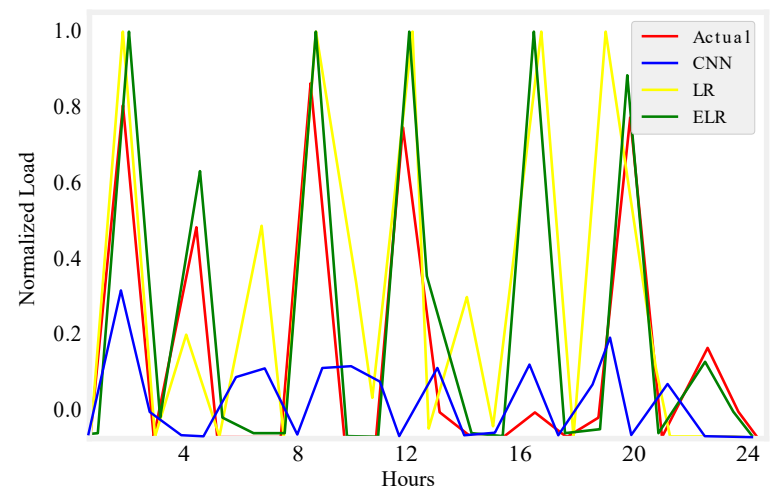

(b) Load prediction using UCI

Figure 5. One day load prediction.

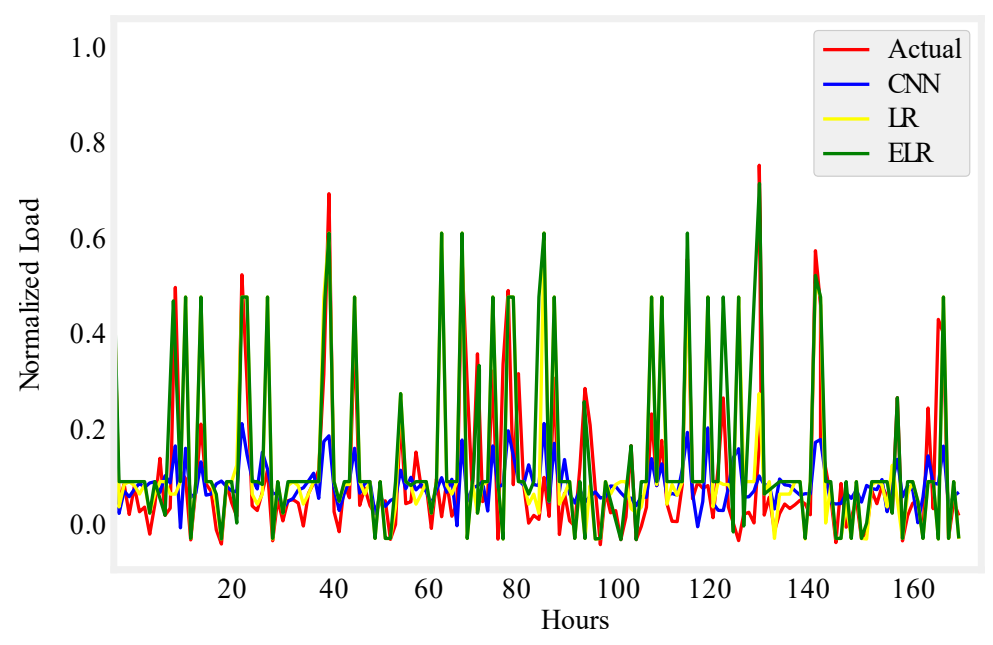

(a) Load prediction using UMass

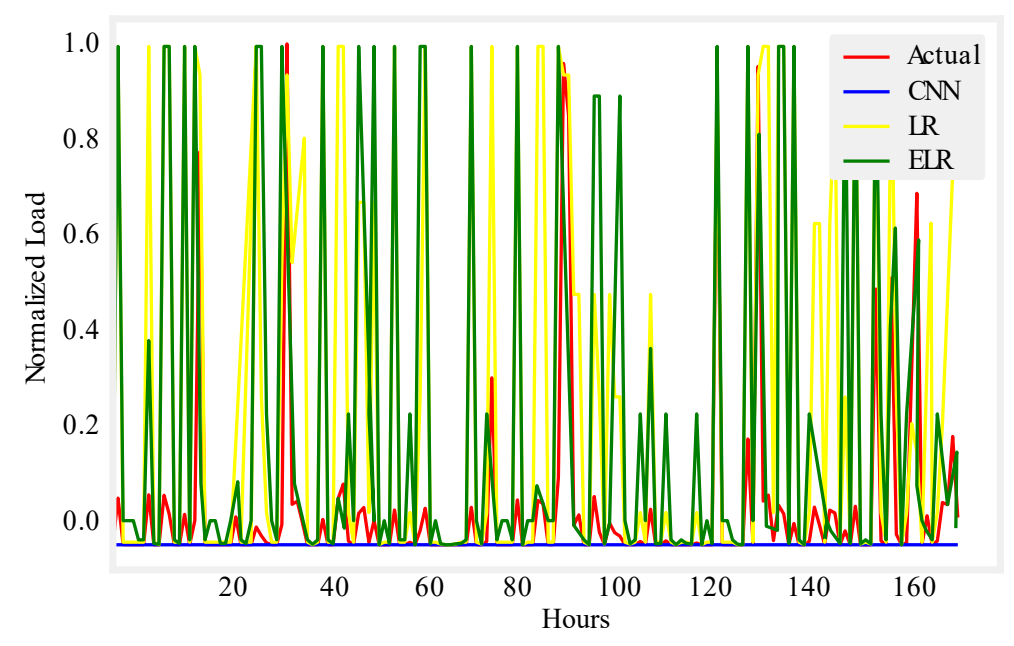

(b) Load prediction using UCI

Figure 6. One week load prediction. 


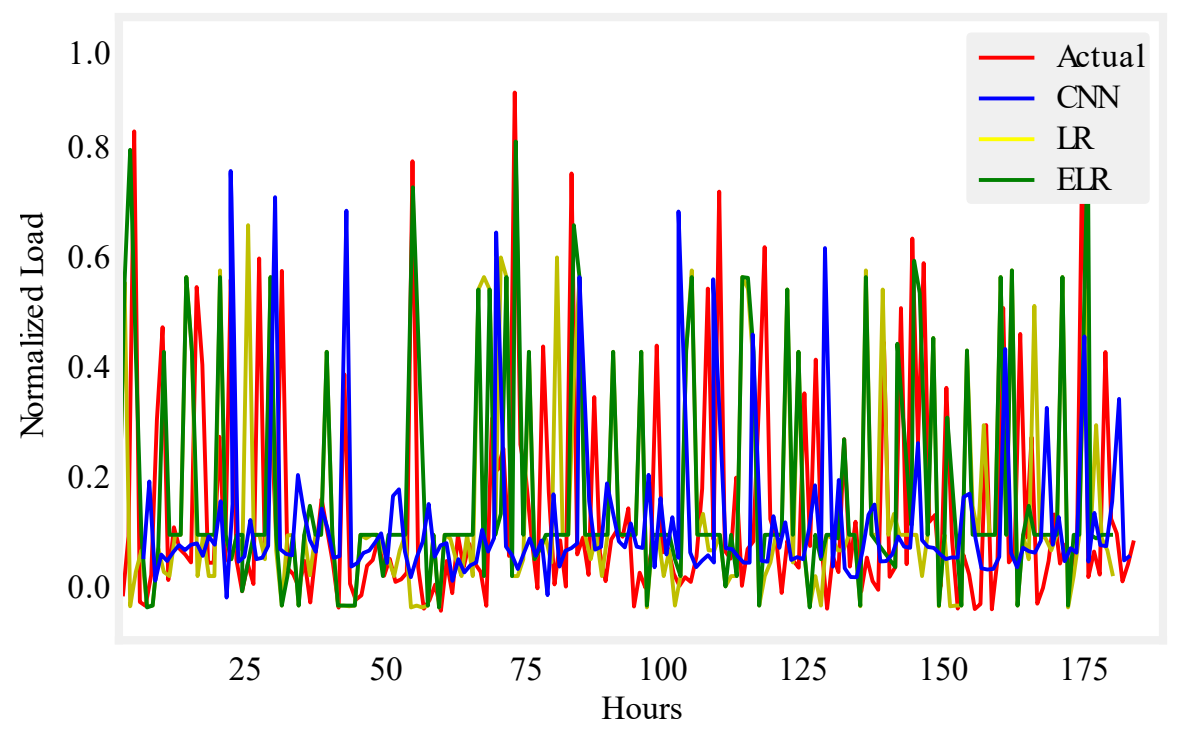

(a) Load prediction using UMass

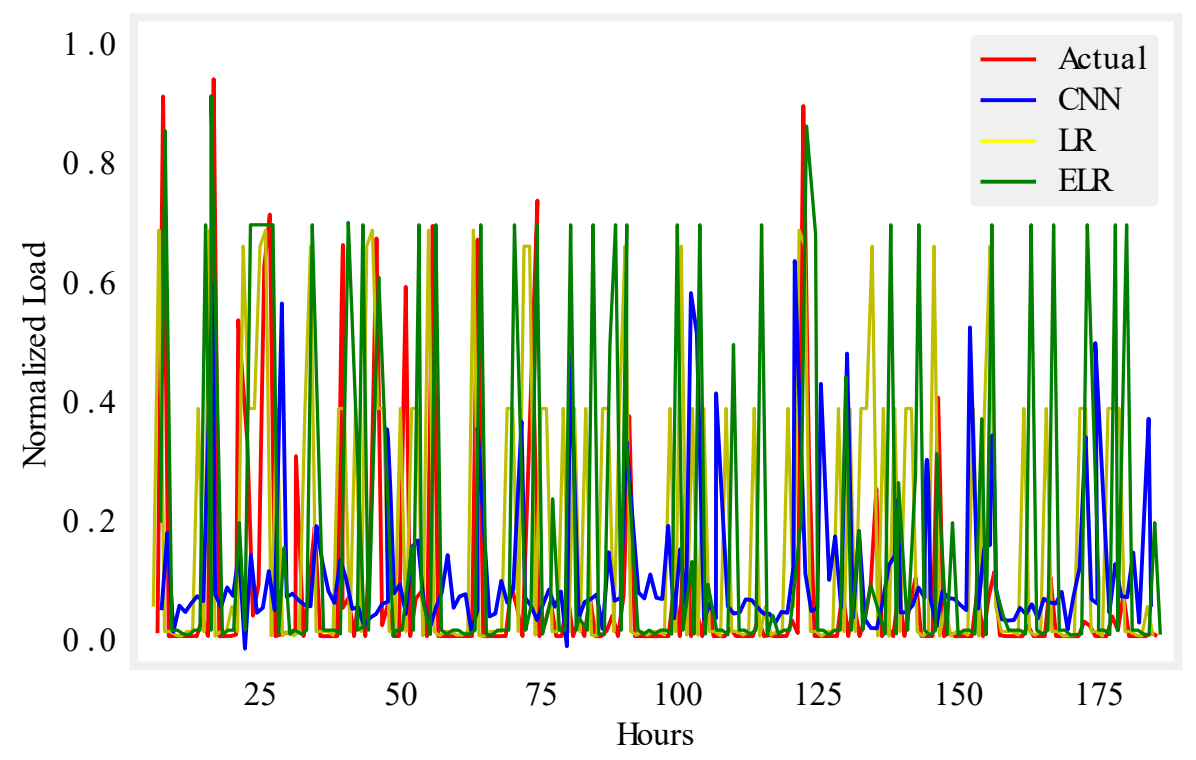

(b) Load prediction using UCI

Figure 7. One month load prediction.

\subsubsection{Price Forecasting}

Figure 8 shows the price prediction comparison of three different techniques for one day using UMass Electric Dataset. Similarly, Figure 9 shows the price prediction comparison for one week using UMass Electric Dataset. From Figure 10, monthly price prediction comparison can be observed. In this case, data is taken every four hours. These figures show that the proposed technique ELR outperformed LR and CNN for UMass Electric Dataset in terms of price prediction. It can be envisioned that the price prediction with ELR is close to the actual data. 


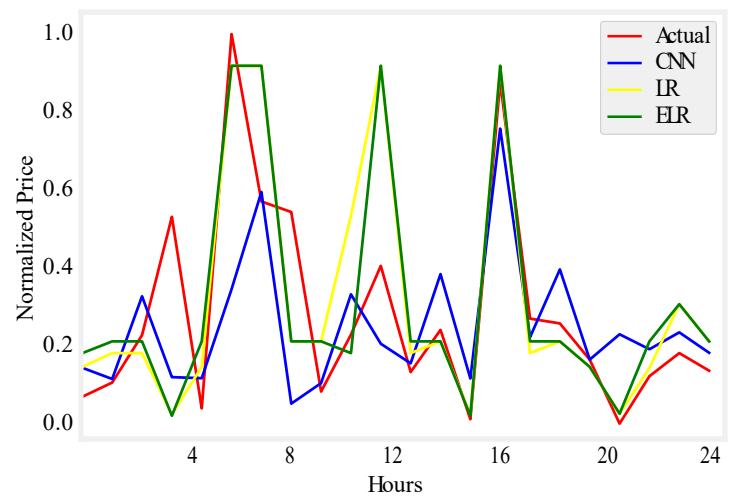

Figure 8. One day price prediction using UMass.

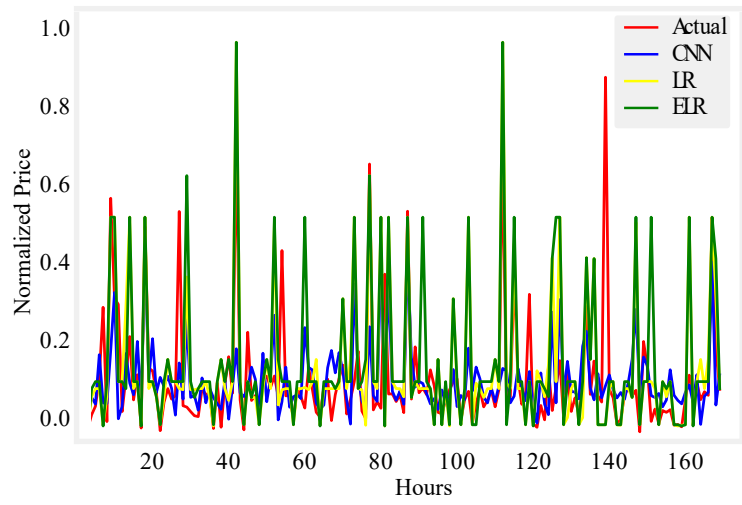

Figure 9. One week price prediction using UMass.

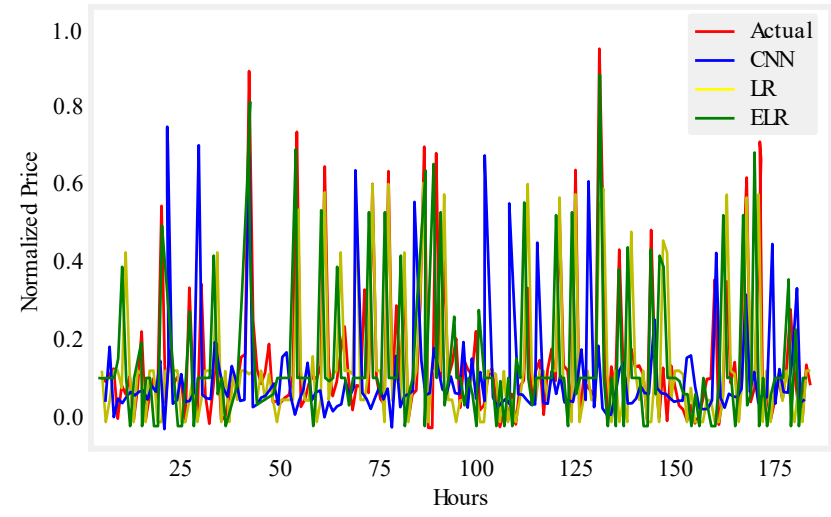

Figure 10. One month price prediction using UMass.

5.2. Simulation Results and Discussion of Proposed System Model 2

The simulation results and discussion of proposed system model 2 are given in this section.

\subsubsection{Data Description}

The second dataset is taken from the UCI machine learning repository. It is a uni-variate dataset developed by Artur Trindade [39]. Consumption of 370 substations is taken under consideration to analyze the load of smart meters. Daily data of meter ID: 166, 168, 169, 171, 182, 225, 237, 249,250 and 257 substation is shown in Figure 11. The periodicity of load consumption can be observed. Pattern of intervals give trend of load consumption that later helps in prediction of future electricity load. 
UCI Dataset is used for both proposed system models. In order to analyze scalability, half-yearly and yearly datasets are used. It performs well for smart meters because the only targeted feature is load. The values of load are given in kilo-Watts. This dataset is also normalized in the range [0-1].
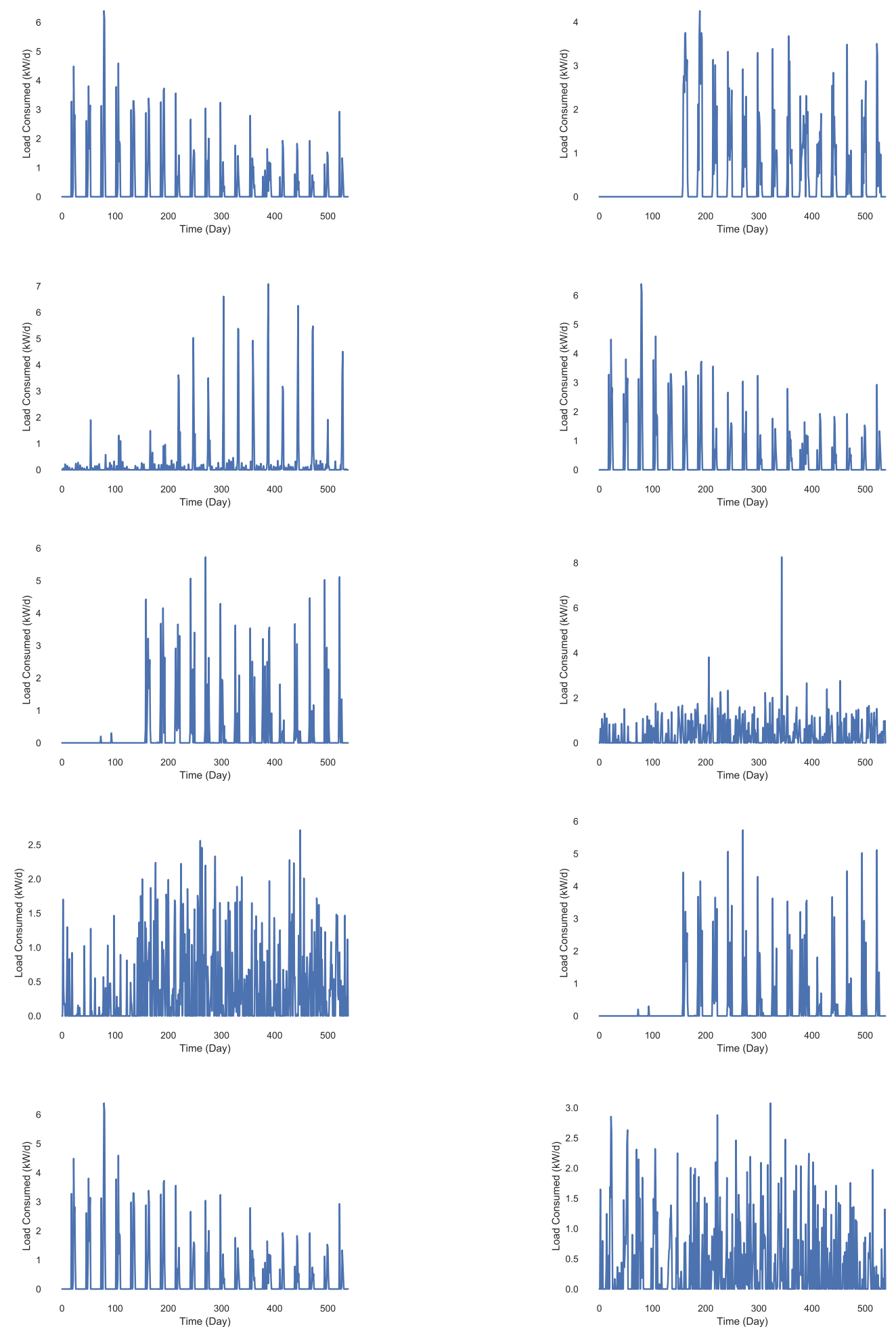

Figure 11. Daily load consumption of 10 different substations. 


\subsubsection{Results Discussion}

Multiple approximation function is used in order to find optimal forecasting accuracy. These functions include hard limit, sine, tanh and sigmoid function. Number of neurons and context neurons are assumed as 2 and 5. The MT166 dataset is selected to finalize functions that are producing optimal results. The dataset is normalized and scaled before use to remove spikes and noise in data. ELM, RELM and ERELM are tested on all functions one by one as given in Table 9. Sigmoid approximation function performed better than other functions. The simulations for the second proposed model are carried out on both datasets using the sigmoid approximation function.

Table 9. Obtained RMSE using ELM, RELM and ERELM.

\begin{tabular}{cccc}
\hline Transfer Function & Forecasting Approach & Training & Testing \\
\hline \multirow{3}{*}{ Hard Limit } & ELM & 0.0532 & 0.0535 \\
& RELM & 0.0412 & 0.0423 \\
& ERELM & 0.0332 & 0.0345 \\
\hline \multirow{3}{*}{ Sin } & ELM & 0.0513 & 0.0525 \\
& RELM & 0.0352 & 0.362 \\
& ERELM & 0.0321 & 0.0523 \\
\hline \multirow{3}{*}{ Tanh } & ELM & 0.0634 & 0.0673 \\
& RELM & 0.0453 & 0.0463 \\
& ERELM & 0.0341 & 0.0321 \\
\hline \multirow{3}{*}{ Sigmoid } & ELM & 0.0423 & 0.473 \\
& RELM & 0.0341 & 0.0381 \\
& ERELM & 0.0214 & 0.0235 \\
\hline
\end{tabular}

In Table 10, simulation results of both datasets are given using six months of data. Cross validation is done using Monte Carlo and K-Fold. Simulations show that the proposed technique outperformed the conventional techniques in perspective of forecasting and gives minimum RMSE. Monte Carlo gives better results as compared to K-Fold. Similarly, Table 11 addresses the scalability of the proposed system and proves that the prediction accuracy increases with the increase in size of dataset. The Figure 12a,b show regression line produced by predicted and actual load using ELM. Similarly, Figure 13a,b show greater RMSE as compared to a proposed technique in regression plot using RELM. Figure 14a,b show plots produced by ERELM, where the regression line shows actual and predicted electricity load with minimum RMSE.

Table 10. Obtained RMSE for half-yearly testing data using ELM, RELM and ERELM by Monte Carlo and $K$-Fold cross validation.

\begin{tabular}{llllllllllll}
\hline \multirow{2}{*}{ Datasets } & \multicolumn{2}{c}{ ERELM } & \multicolumn{2}{c}{ RELM } & \multicolumn{2}{c}{ ELM } & \multicolumn{2}{c}{ RNN } & \multicolumn{2}{c}{ LR } \\
& $\begin{array}{l}\text { Monte } \\
\text { Carlo }\end{array}$ & K-Fold & $\begin{array}{l}\text { Monte } \\
\text { Carlo }\end{array}$ & K-Fold & $\begin{array}{l}\text { Monte } \\
\text { Carlo }\end{array}$ & K-Fold & $\begin{array}{l}\text { Monte } \\
\text { Carlo }\end{array}$ & K-Fold & $\begin{array}{l}\text { Monte } \\
\text { Carlo }\end{array}$ & K-Fold \\
\hline MT166 & 0.0235 & 0.0265 & 0.0734 & 0.0788 & 0.0824 & 0.0883 & 0.08234 & 0.0852 & 0.0853 & 0.0873 \\
MT168 & 0.0134 & 0.0162 & 0.0421 & 0.0462 & 0.0524 & 0.0423 & 0.0854 & 0.0862 & 0.0756 & 0.0763 \\
MT169 & 0.0153 & 0.02352 & 0.0531 & 0.0353 & 0.0382 & 0.0463 & 0.0853 & 0.0873 & 0.0735 & 0.0762 \\
MT171 & 0.0354 & 0.0423 & 0.0524 & 0.0552 & 0.0634 & 0.0643 & 0.0854 & 0.0852 & 0.072 & 0.0712 \\
MT182 & 0.0242 & 0.0353 & 0.0252 & 0.0352 & 0.0835 & 0.0952 & 0.0753 & 0.0776 & 0.0934 & 0.0952 \\
MT235 & 0.0153 & 0.0142 & 0.0344 & 0.0397 & 0.0634 & 0.0643 & 0.0865 & 0.934 & 0.981 & 0.0991 \\
MT237 & 0.0243 & 0.0297 & 0.0534 & 0.0535 & 0.0752 & 0.0795 & 0.0756 & 0.0762 & 0.0795 & 0.08255 \\
MT249 & 0.0143 & 0.0163 & 0.0524 & 0.0532 & 0.0624 & 0.0693 & 0.0862 & 0.0891 & 0.0753 & 0.0778 \\
MT250 & 0.0342 & 0.0452 & 0.0535 & 0.0562 & 0.0853 & 0.0873 & 0.0764 & 0.0784 & 0.0874 & 0.0894 \\
MT257 & 0.0242 & 0.0215 & 0.0413 & 0.0413 & 0.0642 & 0.0683 & 0.0753 & 0.0794 & 0.0893 & 0.0934 \\
UMass Electric & 0.0398 & 0.0315 & 0.0534 & 0.0563 & 0.0681 & 0.0685 & 0.0712 & 0.0891 & 0.0888 & 0.0913 \\
\hline
\end{tabular}


Table 11. Obtained RMSE for yearly testing data using ELM, RELM and ERELM by Monte Carlo and $K$-Fold cross validation.

\begin{tabular}{|c|c|c|c|c|c|c|c|c|c|c|}
\hline \multirow[b]{2}{*}{ Datasets } & \multicolumn{2}{|c|}{ ERELM } & \multicolumn{2}{|c|}{ RELM } & \multicolumn{2}{|c|}{ ELM } & \multicolumn{2}{|c|}{ RNN } & \multicolumn{2}{|c|}{ LR } \\
\hline & $\begin{array}{l}\text { Monte } \\
\text { Carlo }\end{array}$ & K-Fold & $\begin{array}{l}\text { Monte } \\
\text { Carlo }\end{array}$ & K-Fold & $\begin{array}{l}\text { Monte } \\
\text { Carlo }\end{array}$ & K-Fold & $\begin{array}{l}\text { Monte } \\
\text { Carlo }\end{array}$ & K-Fold & $\begin{array}{l}\text { Monte } \\
\text { Carlo }\end{array}$ & $K$-Fold \\
\hline MT166 & 0.0224 & 0.0242 & 0.0651 & 0.0665 & 0.0756 & 0.0801 & 0.08732 & 0.0792 & 0.0862 & 0.0851 \\
\hline MT168 & 0.0124 & 0.0151 & 0.0634 & 0.0732 & 0.0521 & 0.0410 & 0.0831 & 0.0731 & 0.0701 & 0.0678 \\
\hline MT169 & 0.0144 & 0.0224 & 0.0501 & 0.0553 & 0.0424 & 0.0431 & 0.0812 & 0.0912 & 0.0741 & 0.0872 \\
\hline MT171 & 0.0142 & 0.0401 & 0.0421 & 0.0538 & 0.0512 & 0.0682 & 0.0781 & 0.0792 & 0.0712 & 0.0882 \\
\hline MT182 & 0.0182 & 0.0200 & 0.0242 & 0.0250 & 0.0743 & 0.0791 & 0.0824 & 0.0701 & 0.0892 & 0.0822 \\
\hline MT235 & 0.0142 & 0.0152 & 0.0301 & 0.0362 & 0.0582 & 0.0602 & 0.0852 & 0.0892 & 0.0889 & 0.0986 \\
\hline MT237 & 0.0224 & 0.0267 & 0.0513 & 0.0521 & 0.0623 & 0.0632 & 0.0701 & 0.0789 & 0.0862 & 0.0813 \\
\hline MT249 & 0.0132 & 0.0157 & 0.0421 & 0.0613 & 0.0523 & 0.0623 & 0.0782 & 0.0802 & 0.0671 & 0.0742 \\
\hline MT250 & 0.0242 & 0.0273 & 0.0412 & 0.0501 & 0.0602 & 0.0692 & 0.0682 & 0.0772 & 0.0785 & 0.0864 \\
\hline MT257 & 0.0324 & 0.0472 & 0.0401 & 0.0513 & 0.0602 & 0.0744 & 0.0623 & 0.0702 & 0.0876 & 0.0882 \\
\hline UMass Electric & 0.0332 & 0.0412 & 0.0542 & 0.0552 & 0.0582 & 0.0603 & 0.0701 & 0.0771 & 0.0821 & 0.0891 \\
\hline
\end{tabular}

It is clearly visible that predicted values are very close to the actual electricity load. Table 12 gives computational time comparison for execution of training and testing data of ELM, RELM and ERELM. ERELM has great computational time as compared to ELM and RELM due to its metaheuristic behaviour. Thus, there is a tradeoff between accuracy and computational time.

Table 12. Computational time comparison of ERELM, RELM and ELM execution.

\begin{tabular}{ccc}
\hline Forecasting Technique & Training Time (s) & Testing Time (s) \\
\hline ERELM & 0.653 & 0.0346 \\
RELM & 0.0043 & 0.0012 \\
ELM & 0.00124 & 0.001076 \\
\hline
\end{tabular}

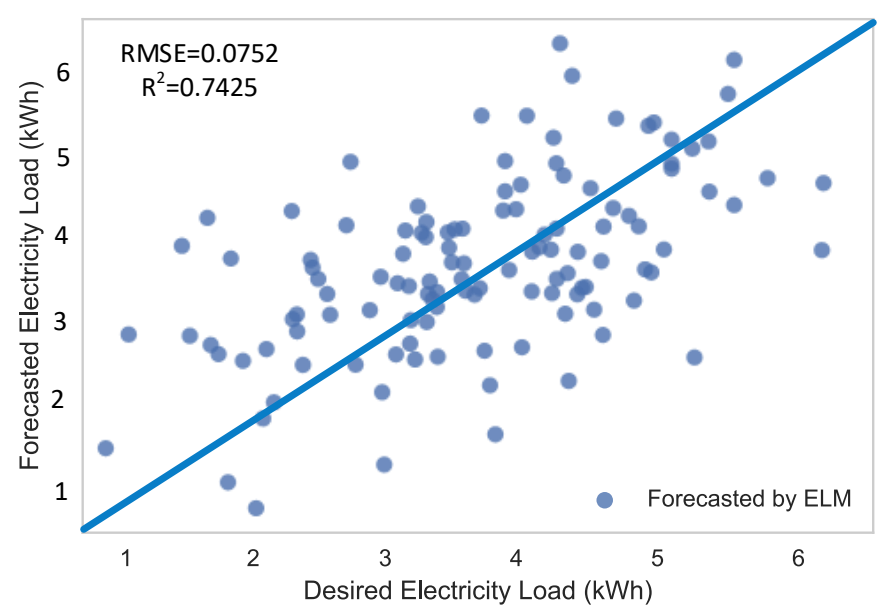

(a) ELM regression line plot for UMass

Figure 12. Cont. 


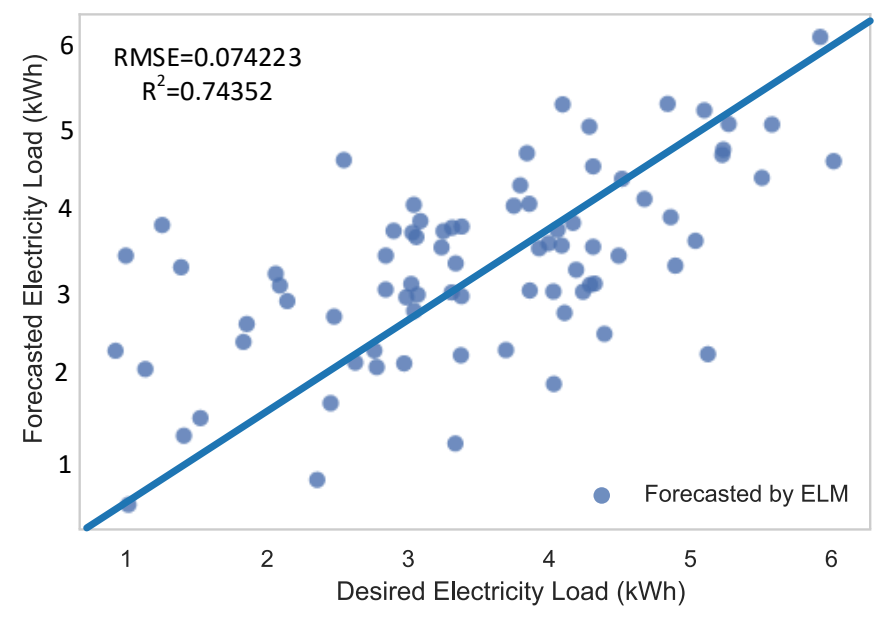

(b) ELM regression line plot for UCI

Figure 12. Regression line plots using ELM.

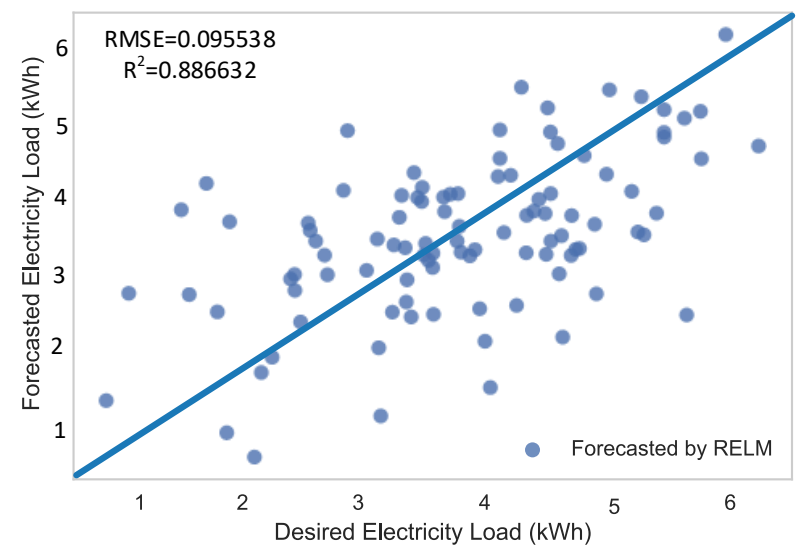

(a) RELM regression line plot for UMass

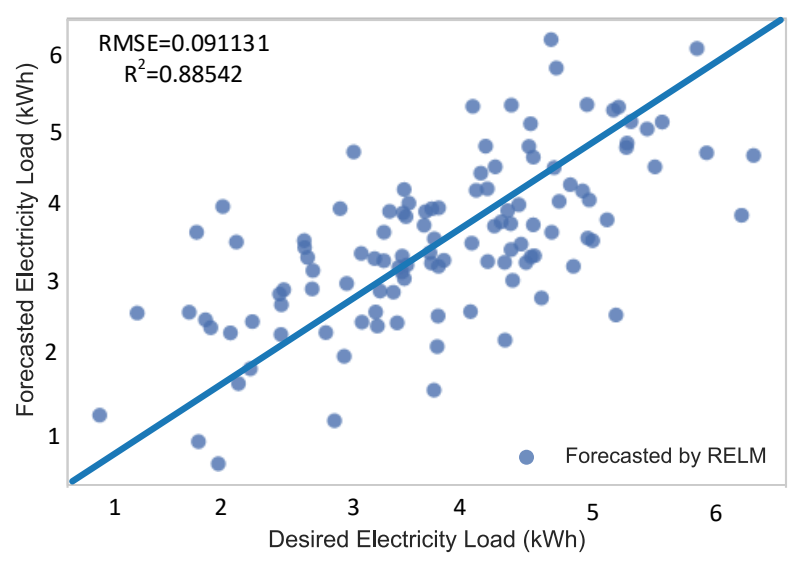

(b) RELM regression line plot for UCI

Figure 13. Regression line plots using RELM. 


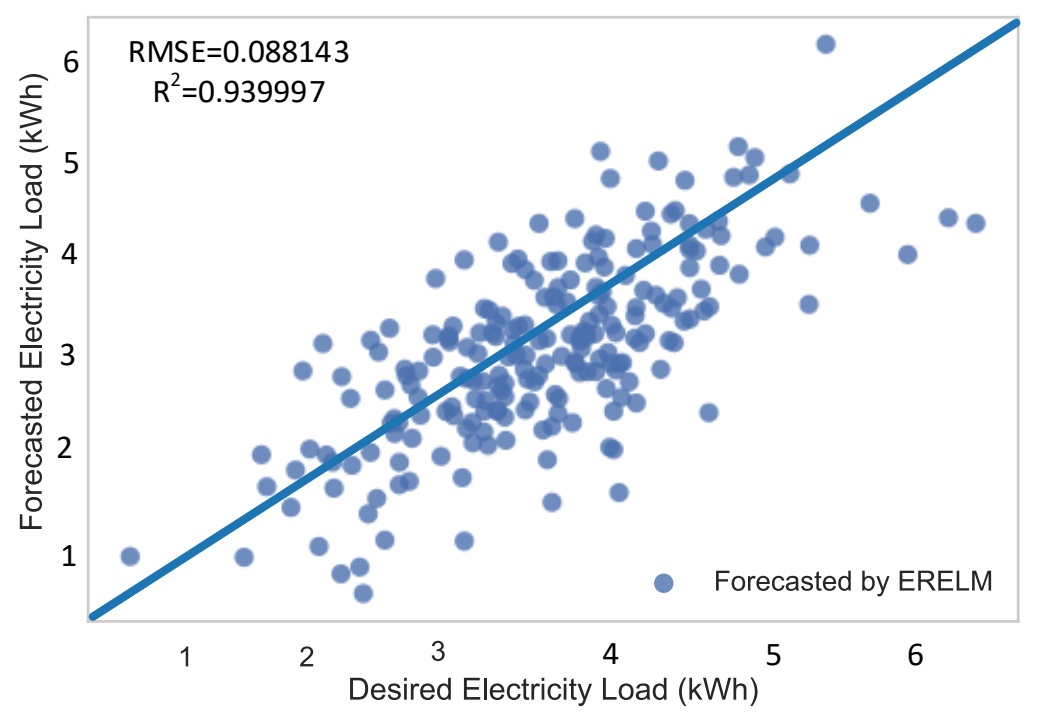

(a) ERELM regression line plot for UMass

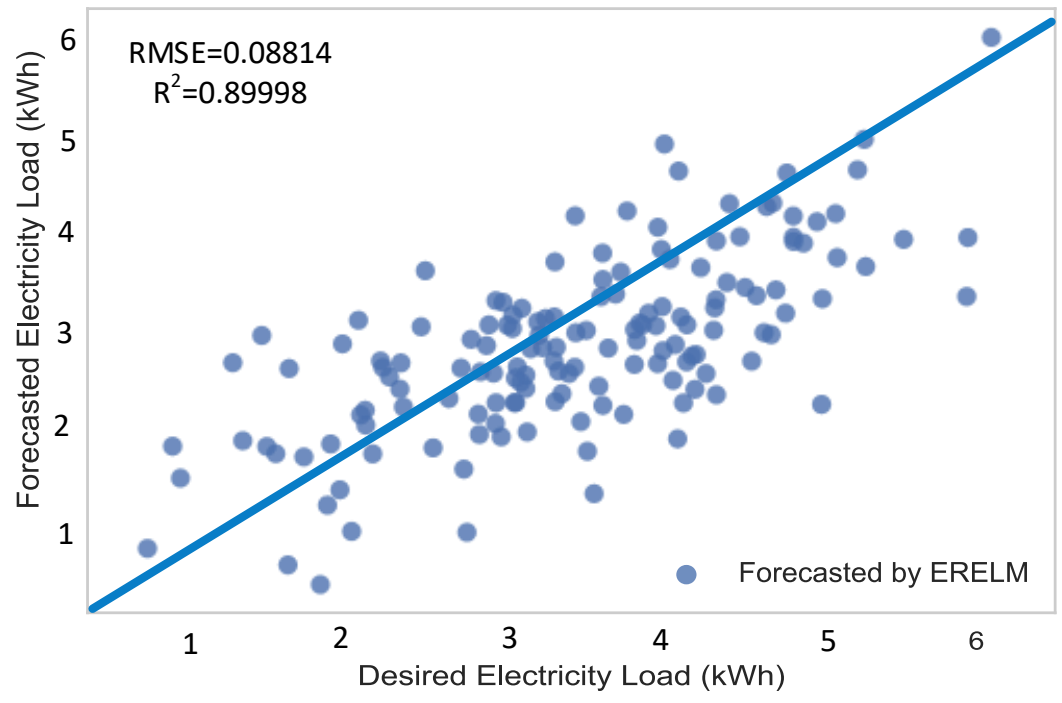

(b) ERELM regression line plot for UCI

Figure 14. Regression line plots using ERELM.

\section{Performance Metrics}

The performance of the proposed system models is evaluated on basis of four performance metrics. These performance metrics are: MAE, MSE, RMSE and MAPE. Out of these four, MAPE is given in terms of percentage whereas, the other three are given as absolute values:

$$
M A P E=\frac{1}{T} \sum_{t m=1}^{T M}\left|\frac{A_{v}}{F_{v}}\right| * 100,
$$




$$
\begin{aligned}
\text { RMSE } & =\sqrt{\frac{1}{T} \sum_{t m=1}^{T M}\left(A_{v}-F_{v}\right)^{2},} \\
M S E & =\frac{1}{T} \sum_{t m=1}^{T M}\left(A_{v}-F_{v}\right)^{2}, \\
M A E & =\frac{\sum_{n=1}^{N}\left|\left(F_{v}-A_{v}\right)\right|}{N} .
\end{aligned}
$$

The accuracy of the model is calculated using the following equation:

$$
\text { Accuracy }=100-\text { RMSE. }
$$

Tables 13-15 show the load performance metrics comparison for half-yearly and yearly data to address the scalability issue. The dataset being used is UMass Electric Dataset. Similarly, Tables 16-18 show the price performance metrics comparison for half-yearly and yearly data to address the scalability issue using the UMass Electric Dataset.

Table 13. Load performance metrics comparison for one day using the UMass Electric Dataset.

\begin{tabular}{lllllll}
\hline \multirow{2}{*}{ Metrics } & \multicolumn{3}{c}{ Half-Yearly Data } & \multicolumn{3}{c}{ Yearly Data } \\
& CNN & LR & ELR & CNN & LR & ELR \\
\hline MSE (abs. val) & 8.84 & 5.84 & 4.77 & 10.2 & 8.97 & 6.8 \\
MAE (abs. val) & 9.24 & 5.25 & 4.34 & 8.75 & 6.25 & 4.24 \\
RMSE (abs. val) & 10.62 & 7.64 & 6.18 & 10.4 & 6.6 & 5.2 \\
MAPE (\%) & 25.44 & 22.45 & 18.48 & 36.3 & 33.3 & 30.5 \\
Accuracy (\%) & 89.38 & 92.35 & 93.82 & 89.6 & 93.4 & 94.8 \\
\hline
\end{tabular}

Table 14. Load performance metrics comparison for one week using the UMass Electric Dataset.

\begin{tabular}{lllllll}
\hline \multirow{2}{*}{ Metrics } & \multicolumn{3}{c}{ Half-Yearly Data } & \multicolumn{3}{c}{ Yearly Data } \\
& CNN & LR & ELR & CNN & LR & ELR \\
\hline MSE (abs. val) & 20.2 & 17.97 & 12.97 & 18.9 & 16.5 & 11.3 \\
MAE (abs. val) & 8.82 & 6.87 & 5.28 & 8.75 & 6.25 & 5.19 \\
RMSE (abs. val) & 16.25 & 13.12 & 10.18 & 15.76 & 12.98 & 9.64 \\
MAPE (\%) & 25.65 & 22.19 & 17.41 & 33.2 & 25.9 & 22.8 \\
Accuracy (\%) & 83.75 & 86.88 & 89.81 & 84.24 & 87.02 & 91.36 \\
\hline
\end{tabular}

Table 15. Load performance metrics comparison for one month using the UMass Electric Dataset.

\begin{tabular}{lllllll}
\hline \multirow{2}{*}{ Metrics } & \multicolumn{3}{c}{ Half-Yearly Data } & \multicolumn{3}{c}{ Yearly Data } \\
& CNN & LR & ELR & CNN & LR & ELR \\
\hline MSE (abs. val) & 25.82 & 21.82 & 17.37 & 24.02 & 20.56 & 14.25 \\
MAE (abs. val) & 10.55 & 8.23 & 6.79 & 10.35 & 8.15 & 5.33 \\
RMSE (abs. val) & 17.85 & 14.77 & 11.79 & 12.55 & 9.98 & 6.52 \\
MAPE (\%) & 29.13 & 27.13 & 23.39 & 25.45 & 21.2 & 20.6 \\
Accuracy (\%) & 82.15 & 85.72 & 88.21 & 87.45 & 90.02 & 93.48 \\
\hline
\end{tabular}


Table 16. Price performance metrics comparison for one day using the UMass Electric Dataset.

\begin{tabular}{lllllll}
\hline \multirow{2}{*}{ Metrics } & \multicolumn{3}{c}{ Half-Yearly Data } & \multicolumn{3}{c}{ Yearly Data } \\
& CNN & LR & ELR & CNN & LR & ELR \\
\hline MSE (abs. val) & 15.08 & 14.39 & 10.09 & 12.58 & 10.85 & 8.6 \\
MAE (abs. val) & 8.25 & 7.65 & 6.01 & 7.85 & 7.05 & 5.88 \\
RMSE (abs. val) & 16.63 & 14.99 & 12.98 & 15.05 & 11.52 & 9.85 \\
MAPE (\%) & 19.02 & 18.59 & 17.11 & 20.05 & 18.85 & 15.55 \\
Accuracy (\%) & 83.37 & 85.01 & 87.02 & 84.95 & 88.48 & 90.15 \\
\hline
\end{tabular}

Table 17. Price performance metrics comparison for one week using the UMass Electric Dataset.

\begin{tabular}{lllllll}
\hline \multirow{2}{*}{ Metrics } & \multicolumn{3}{c}{ Half-Yearly Data } & \multicolumn{3}{c}{ Yearly Data } \\
& CNN & LR & ELR & CNN & LR & ELR \\
\hline MSE (abs. val) & 14.05 & 12.80 & 11.22 & 15.02 & 13.45 & 11.25 \\
MAE (abs. val) & 7.55 & 6.04 & 5.03 & 8.02 & 7.05 & 5.25 \\
RMSE (abs. val) & 13.05 & 11.30 & 9.47 & 12.55 & 10.45 & 8.64 \\
MAPE (\%) & 14.25 & 13.71 & 13.03 & 16.45 & 15.75 & 15.25 \\
Accuracy (\%) & 86.95 & 88.70 & 90.53 & 87.45 & 89.55 & 91.36 \\
\hline
\end{tabular}

Table 18. Price performance metrics comparison for one month using the UMass Electric Dataset.

\begin{tabular}{lllllll}
\hline \multirow{2}{*}{ Metrics } & \multicolumn{3}{c}{ Half-Yearly Data } & \multicolumn{3}{c}{ Yearly Data } \\
& CNN & LR & ELR & CNN & LR & ELR \\
\hline MSE (abs. val) & 19.45 & 18.91 & 16.47 & 20.05 & 18.54 & 13.35 \\
MAE (abs. val) & 8.95 & 7.70 & 6.44 & 9.35 & 8.15 & 6.42 \\
RMSE (abs. val) & 14.78 & 13.75 & 11.48 & 12.44 & 11.02 & 9.45 \\
MAPE (\%) & 21.44 & 20.54 & 18.89 & 23.36 & 22.55 & 19.45 \\
Accuracy (\%) & 85.22 & 86.25 & 88.52 & 87.56 & 88.98 & 90.55 \\
\hline
\end{tabular}

Tables 19-21 show the load performance metrics comparison for half-yearly and yearly data to address the scalability issue. The dataset being used is UCI Dataset.

Table 19. Load performance metrics comparison for one day using the UCI Dataset.

\begin{tabular}{lllllll}
\hline \multirow{2}{*}{ Metrics } & \multicolumn{3}{c}{ Half-Yearly Data } & \multicolumn{3}{c}{ Yearly Data } \\
& CNN & LR & ELR & CNN & LR & ELR \\
\hline MSE (abs. val) & 15.05 & 12.47 & 10.56 & 18.20 & 14.3 & 10.4 \\
MAE (abs. val) & 12.45 & 10.34 & 8.56 & 21.47 & 18.37 & 16.61 \\
RMSE (abs. val) & 18.52 & 15.54 & 13.45 & 16.22 & 13.78 & 10.16 \\
MAPE (\%) & 28.24 & 25.34 & 20.45 & 27.05 & 23.25 & 20.05 \\
Accuracy (\%) & 81.48 & 84.46 & 86.55 & 83.78 & 86.22 & 89.84 \\
\hline
\end{tabular}

Table 20. Load performance metrics comparison for one week using the UCI Dataset.

\begin{tabular}{lllllll}
\hline \multirow{2}{*}{ Metrics } & \multicolumn{3}{c}{ Half-Yearly Data } & \multicolumn{3}{c}{ Yearly Data } \\
& CNN & LR & ELR & CNN & LR & ELR \\
\hline MSE (abs. val) & 25.20 & 19.66 & 15.77 & 13.25 & 8.34 & 7.2 \\
MAE (abs. val) & 11.35 & 10.01 & 8.24 & 13.98 & 12.47 & 11.39 \\
RMSE (abs. val) & 22.45 & 19.25 & 16.45 & 20.25 & 17.80 & 13.11 \\
MAPE (\%) & 28.56 & 25.45 & 19.63 & 30.50 & 25.45 & 18.52 \\
Accuracy (\%) & 77.55 & 80.75 & 83.55 & 79.75 & 82.20 & 86.89 \\
\hline
\end{tabular}


Table 21. Load performance metrics comparison for one month using the UCI Dataset.

\begin{tabular}{lllllll}
\hline \multirow{2}{*}{ Metrics } & \multicolumn{3}{c}{ Half-Yearly Data } & \multicolumn{3}{c}{ Yearly Data } \\
& CNN & LR & ELR & CNN & LR & ELR \\
\hline MSE (abs. val) & 28.35 & 25.55 & 21.68 & 15.50 & 13.8 & 5.47 \\
MAE (abs. val) & 15.35 & 10.34 & 8.95 & 23.46 & 19.3 & 17.7 \\
RMSE (abs. val) & 23.97 & 20.87 & 17.69 & 20.02 & 17.17 & 14.49 \\
MAPE (\%) & 31.23 & 29.43 & 25.67 & 27.45 & 25.35 & 20.02 \\
Accuracy (\%) & 76.03 & 79.13 & 82.31 & 79.98 & 82.23 & 85.51 \\
\hline
\end{tabular}

Tables 22 and 23 represent accuracy of proposed technique ERELM using RMSE, MSE and MAE, using half-yearly and yearly data. Results represent that ERELM outperformed in all performance metrics.

Table 22. Accuracy of ERELM using RMSE, MSE and MAE for half-yearly data.

\begin{tabular}{cccc}
\hline Datasets & RMSE & MSE & MAE \\
\hline MT166 & 0.0235 & 0.00055 & 0.0243 \\
MT168 & 0.0134 & 0.00017 & 0.0135 \\
MT169 & 0.0153 & 0.00023 & 0.0174 \\
MT171 & 0.0354 & 0.00125 & 0.0352 \\
MT182 & 0.0242 & 0.00058 & 0.0252 \\
MT235 & 0.0153 & 0.00023 & 0.0253 \\
MT237 & 0.0243 & 0.00059 & 0.0254 \\
MT249 & 0.0143 & 0.00020 & 0.0153 \\
MT250 & 0.0342 & 0.001169 & 0.0342 \\
MT257 & 0.0242 & 0.00058 & 0.0253 \\
UMass Electric & 0.0256 & 0.00071 & 0.0623 \\
Arithmetic Mean & 0.0227 & 0.00055 & 0.024218 \\
Standard Deviation & 0.00600 & 0.000350 & 0.006515 \\
\hline
\end{tabular}

Table 23. Accuracy of ERELM using RMSE, MSE and MAE for yearly data.

\begin{tabular}{cccc}
\hline Datasets & RMSE & MSE & MAE \\
\hline MT166 & 0.0224 & 0.00041 & 0.0215 \\
MT168 & 0.0124 & 0.00016 & 0.0142 \\
MT169 & 0.0144 & 0.00015 & 0.0162 \\
MT171 & 0.0142 & 0.00124 & 0.0221 \\
MT182 & 0.0200 & 0.00042 & 0.0224 \\
MT235 & 0.0142 & 0.00047 & 0.0201 \\
MT237 & 0.0224 & 0.00015 & 0.0241 \\
MT249 & 0.0132 & 0.00012 & 0.0142 \\
MT250 & 0.0242 & 0.00102 & 0.0163 \\
MT257 & 0.0324 & 0.00045 & 0.0177 \\
UMass Electric & 0.0332 & 0.00061 & 0.0546 \\
Arithmetic Mean & 0.02027 & 0.00047 & 0.02212 \\
Standard Deviation & 0.00712 & 0.00034 & 0.01077 \\
\hline
\end{tabular}

\section{Conclusions and Future Work}

In this paper, electricity load and price forecasting are performed using two techniques. UMass Electric Dataset is used to predict day ahead, week ahead and month ahead load and price of a SH. Six months of hourly data are considered for day ahead and week ahead prediction, whereas four hours of data are considered for month ahead prediction. It is a multi-variate dataset. The data is first normalized and split into a training set and testing set. Feature engineering is then performed using three different techniques: RFE, CART and Relief-F. For efficient load and price prediction, a new technique, i.e., ELR is proposed. ELR outperformed CNN and LR in terms of prediction accuracy. ELR is used 
for UCI Dataset as well. It is a uni-variate dataset having data of smart meters of different substations. The results show that the first proposed model works well with UMass Electric Dataset. The techniques used are then accessed on the basis of four different performance metrics, i.e., MAPE, MAE, MSE and RMSE. The simulation results show that ELR outperformed LR and CNN for both datasets.

For accurate short term load forecasting, a new technique, i.e., ERELM is proposed. Short term forecasting is performed to ensure efficient load scheduling and price reduction. Parameter optimization of RELM is done using GWO. GWO optimizes biases and weights to improve the accuracy. Prediction accuracy is further increased using Monte Carlo and K-Fold. ERELM is used with both datasets. The results show that ERELM works well for UCI Datasets. It is observed that ERELM outperformed ELM and RELM for both datasets. The phenomenon of scalability is also addressed using both proposed techniques. Results prove that the prediction accuracy increases with the increase in size of dataset.

In future, the proposed methods will be used to perform mid-term and long-term forecasting. Weights and biases of ERELM will be further optimized using better methods. Furthermore, efficient work is required to reduce the computational time of ELR and ERELM.

Author Contributions: All authors contributed equally.

Acknowledgments: The authors extend their appreciation to the Deanship of Scientific Research at King Saud University for funding this work through research group NO (RG-1438-034).

Conflicts of Interest: The authors declare no conflicts of interest.

\section{References}

1. Ipakchi, A.; Albuyeh, F. Grid of the future. IEEE Power Energy Mag. 2009, 7, 52-62. [CrossRef]

2. Yoldaş, Y.; Önen, A.; Muyeen, S.M.; Vasilakos, A.V.; Alan, İ. Enhancing smart grid with microgrids: Challenges and opportunities. Renew. Sustain. Energy Rev. 2017, 72, 205-214. [CrossRef]

3. Shaukat, N.; Ali, S.M.; Mehmood, C.A.; Khan, B.; Jawad, M.; Farid, U.; Ullah, Z.; Anwar, S.M.; Majid, M. A survey on consumers empowerment, communication technologies, and renewable generation penetration within Smart Grid. Renew. Sustain. Energy Rev. 2018, 81, 1453-1475. [CrossRef]

4. Zhou, K.; Fu, C.; Yang, S. Big data driven smart energy management: From big data to big insights. Renew. Sustain. Energy Rev. 2016, 56, 215-225. [CrossRef]

5. Nazar, M.S.; Fard, A.E.; Heidari, A.; Shafie-khah, M.; Catalão, J.P.S. Hybrid model using three-stage algorithm for simultaneous load and price forecasting. Electr. Power Syst. Res. 2018, 165, 214-228. [CrossRef]

6. Ertugrul, Ö.F. Forecasting electricity load by a novel recurrent extreme learning machines approach. Int. J. Electr. Power Energy Syst. 2016, 78, 429-435. [CrossRef]

7. Muralitharan, K.; Sakthivel, R.; Vishnuvarthan, R. Neural network based optimization approach for energy demand prediction in smart grid. Neurocomputing 2018, 273, 199-208. [CrossRef]

8. Shailendra, S.; Yassine, A. Big Data Mining of Energy Time Series for Behavioral Analytics and Energy Consumption Forecasting. Energies 2018, 11, 452. [CrossRef]

9. Ahmad, T.; Chen, H. Short and medium-term forecasting of cooling and heating load demand in building environment with data-mining based approaches. Energy Build. 2018, 166, 460-476. [CrossRef]

10. Kunjin, C.; Kunlong, C.; Qin, W.; Ziyu, H.; Jun, H.; He, J. Short-term Load Forecasting with Deep Residual Networks. IEEE Trans. Smart Grid 2018, 99. [CrossRef]

11. Seunghyoung, R.; Noh, J.; Kim, H. Deep neural network based demand side short term load forecasting. Energies 2016, 10, 3 .

12. Liu, J.P.; Li, C.L. The short-term power load forecasting based on sperm whale algorithm and wavelet least square support vector machine with DWT-IR for feature selection. Sustainability 2017, 9, 1188. [CrossRef]

13. Ahmad, A.; Javaid, N.; Guizani, M.; Alrajeh, N.; Khan, Z.A. An accurate and fast converging short-term load forecasting model for industrial applications in a smart grid. IEEE Trans. Ind. Inform. 2017, 13, 2587-2596. [CrossRef]

14. Fan, C.; Xiao, F.; Zhao, Y. A short-term building cooling load prediction method using deep learning algorithms. Appl. Energy 2017, 195, 222-233. [CrossRef] 
15. Shi, H.; Xu, M.; Li, R. Deep learning for household load forecasting-A novel pooling deep RNN. IEEE Trans. Smart Grid 2018, 9, 5271-5280. [CrossRef]

16. Huang, G.B.; Zhu, Q.Y.; Siew, C.K. Extreme learning machine: theory and applications. Neurocomputing 2006, 70, 489-501. [CrossRef]

17. Huang, G.B.; Zhou, H.; Ding, X.; Zhang, R. Extreme learning machine for regression and multiclass classification. IEEE Trans. Syst. Man Cybern. Part B 2012, 42, 513-529. [CrossRef] [PubMed]

18. Fallah, S.N.; Deo, R.C.; Shojafar, M.; Conti, M.; Shamshirband, S. Computational Intelligence Approaches for Energy Load Forecasting in Smart Energy Management Grids: State of the Art, Future Challenges, and Research Directions. Energies 2018, 11, 596. [CrossRef]

19. Zeng, Y.R.; Zeng, Y.; Choi, B.; Wang, L. Multifactor-influenced energy consumption forecasting using enhanced back-propagation neural network. Energy 2018, 127, 381-396. [CrossRef]

20. Luo, J.; Vong, C.M.; Wong, P.K. Sparse Bayesian extreme learning machine for multi-classification. IEEE Trans. Neural Netw. Learn. Syst. 2014, 25, 836-843. [PubMed]

21. Yu, J.; Wang, S.; Xi, L. Evolving artificial neural networks using an improved PSO and DPSO. Neurocomputing 2008, 71, 1054-1060. [CrossRef]

22. Mirjalili, S.; Mirjalili, S.M.; Lewis, A. Grey wolf optimizer. Adv. Eng. Softw. 2014, 69, 46-61. [CrossRef]

23. Saremi, S.; Mirjalili, S.Z.; Mirjalili, S.M. Evolutionary population dynamics and grey wolf optimizer. Neural Comput. Appl. 2015, 26, 1257-1263. [CrossRef]

24. Lago, J.; De Ridder, F.; De Schutter, B. Forecasting spot electricity prices: deep learning approaches and empirical comparison of traditional algorithms. Appl. Energy 2018, 221, 386-405. [CrossRef]

25. González, J.P.; San Roque, A.M.; Perez, E.A. Forecasting functional time series with a new Hilbertian ARMAX model: Application to electricity price forecasting. IEEE Trans. Power Syst. 2018, 33, 545-556. [CrossRef]

26. Kuo, P.H.; Huang, C.J. An Electricity Price Forecasting Model by Hybrid Structured Deep Neural Networks. Sustainability 2018, 10, 1280. [CrossRef]

27. Wang, K.; Xu, C.; Zhang, Y.; Guo, S.; Zomaya, A. Robust big data analytics for electricity price forecasting in the smart grid. IEEE Trans. Big Data 2017, 5, 34-45. [CrossRef]

28. Lago, J.; De Ridder, F.; Vrancx, P.; De Schutter, B. Forecasting day-ahead electricity prices in Europe: the importance of considering market integration. Appl. Energy 2018, 211, 890-903. [CrossRef]

29. Long, W.; Zhang, Z.; Chen, J. Short-Term Electricity Price Forecasting with Stacked Denoising Autoencoders. IEEE Trans. Power Syst. 2017, 32, 2673-2681.

30. Ghasemi, A.; Shayeghi, H.; Moradzadeh, M.; Nooshyar, M. A novel hybrid algorithm for electricity price and load forecasting in smart grids with demand-side management. Appl. Energy 2016, 177, 40-59. [CrossRef]

31. Huang, G.B.; Chen, L.; Siew, C.K. Universal approximation using incremental constructive feedforward networks with random hidden nodes. IEEE Trans. Neural Netw. 2006, 17, 879-892. [CrossRef] [PubMed]

32. Bartlett, P.L. For valid generalization the size of the weights is more important than the size of the network. In Advances in Neural Information Processing Systems; MIT Press: Cambridge, MA, USA, 1997; pp. 134-140.

33. Scardapane, S.; Comminiello, D.; Scarpiniti, M.; Uncini, A. Online sequential extreme learning machine with kernels. IEEE Trans. Neural Netw. Learn. Syst. 2015, 26, 2214-2220. [CrossRef] [PubMed]

34. Loh, W.Y. Classification and Regression Trees. Wiley Interdisciplinary Reviews: Data Mining and Knowledge Discovery; John Wiley and Sons Inc.: Hoboken, NJ, USA, 2011; Volume 1, pp. 14-23.

35. Recursive Feature Elimination. Available online: https://topepo.github.io/caret/recursive-featureelimination.html (accessed on 10 November 2018).

36. Durgabai, R.P.L. Feature selection using ReliefF algorithm. Int. J. Adv. Res. Comput. Commun. Eng. 2014, 3, 10, 8215-8218.

37. Logistic Regression. Available online: https://ml-cheatsheet.readthedocs.io/en/latest/logistic-regression. html (accessed on 10 November 2018).

38. UMass Electric Dataset. Available online: http://traces.cs.umass.edu/index.php/Smart/Smart (accessed on 10 November 2018).

39. Lichman, M. UCI Machine Learning Repository; University of California: Irvine, CA, USA, 2013.

(C) 2019 by the authors. Licensee MDPI, Basel, Switzerland. This article is an open access article distributed under the terms and conditions of the Creative Commons Attribution (CC BY) license (http:/ / creativecommons.org/licenses/by/4.0/). 